\title{
The effects of free-stream turbulence on the performance of a model wind turbine
}

\author{
Stefano Gambuzza ${ }^{\text {a) }}$ and Bharathram Ganapathisubramani \\ Aerodynamics and Flight Mechanics Group, Faculty of Engineering and Physical Sciences, \\ University of Southampton, Southampton, UK, SO17 1BJ
}

(Dated: 10 March 2021)

Free-stream turbulence characteristics play an important role in the mechanisms of power harvesting for wind turbines. Acquisitions of power and thrust from a model wind turbine of diameter $0.18 \mathrm{~m}$ have been carried out in a wind tunnel for a wide range of turbulent base flows, with varying free-stream turbulence intensity in the range between $3 \%$ and $16 \%$ and integral time scale spanning from 0.1 to 10 times the turbine rotation period. The results demonstrate that power is significantly affected both by the inflow turbulence scales and its intensity, while thrust is scarcely affected by free-stream turbulence. Fluctuations in the generated torques are also measured, with their behaviour dominated by the free-stream turbulence scale, and only moderately affected by turbulence intensity. The frequency response of thrust fluctuations has been measured for a selected subset of operating conditions, demonstrating that the turbine thrust is unaffected by high-frequency components in the inflow. Conclusions are drawn on the necessity to match both turbulence intensity and base flow frequency content in wind-tunnel studies if realistic results are to be obtained from small-scale studies.

Keywords: Free-stream turbulence, Model wind turbine, Power harvesting, Bluffbody drag

\section{INTRODUCTION}

In recent years, electrical power harvested from wind energy sources has been constantly growing, with peaks of $30 \%$ of the United Kingdom power demand being provided from wind and tidal turbines. Wind turbines generate power from winds naturally present in the atmospheric boundary layer, which are characterized by complex turbulent structures, variable both in time and over the rotor swept area. These structures span a large range of scales, from larger meso-scale eddies that are generated by the interaction of the atmospheric boundary layer with the surface to frequent smaller-scale gusts ${ }^{1}$, and cause large fluctuations in power generated by a wind turbine ${ }^{2}$. The interaction of wind turbines with these structures is not straightforward to characterize.

To study how wind turbine performance figures (namely, the thrust generated or the power harvested by the turbine) are affected by different turbulent flows, a wide number of experimental studies have been carried out. These range from experiments with porous $\operatorname{discs}^{3,4}$, to model-scale turbines in wind tunnels or water channels ${ }^{5-7}$, to observations on real-scale turbines generating usable electrical power ${ }^{8-10}$; the last are obviously scarcer due to inherent difficulties in undertaking rigorous measurements in natural environments.

The effect of the oncoming flow on drag experienced by bluff bodies has received considerable attention. Previous studies ${ }^{11}$ identify the free-stream turbulence intensity as the main parameter driving drag variations. For model wind turbines at Reynolds numbers in the order of $10^{5}$ and turbulence intensities under $20 \%$, conflicting results are reported in literature: some studies observe consistent reductions in the thrust generated by model turbines with increasing turbulence intensity ${ }^{5,7}$, while other studies report an increase in thrust for more turbulent flows ${ }^{6}$ or comparable values of velocity deficit in the near wake

a) Electronic mail: s.gambuzza@soton.ac.uk 
(thus, comparable thrust) for different turbulence intensities ${ }^{12}$. It must be noted that none of these studies (and neither does this one) employ a common model wind turbine design, and thus different results can, in principle, be expected; however, such large discrepancies in the general trends cannot be explained by different designs alone, and some other mechanisms previously overlooked could affect these trends. The nature of these mechanisms is not evident from the works currently present in literature: for this reason, we want to highlight the necessity for a thorough parametric study on the effects of different turbulence parameters on a wind turbine thrust to be undertaken, to ascertain whether previously overlooked quantities can explain these conflicting results.

More consistent results are obtained from the investigation of porous and thin bodies in turbulent flows; drawing parallels between these bodies and model-scale turbines is justified by the good match between their wakes, especially at low Reynolds numbers and moderate turbulence intensities ${ }^{13,14}$. In particular, ref. 3 obtains, for discs of different porosity, an increasing drag with free-stream turbulence intensity, with a smaller effect of free-stream integral time scale on the results also present. Similar trends are observed for thin solid bodies perpendicular to the free-stream ${ }^{15,16}$.

Similarly, there is a strong interest in modelling the effects of free-stream turbulence on the power harvested by a wind turbine. For this aspect, some analytical models have been proposed in literature; the perhaps simplest of these is presented by ref. 8 , where power is assumed to increase with the square of free-stream turbulence intensity:

$$
P\left(U_{\infty}, \mathcal{I}_{\sigma}\right)=\frac{1}{2} \rho U_{\infty}^{3}\left(1+3 \mathcal{I}_{\sigma}^{2}\right) \pi R^{2} C_{P}
$$

for a wind turbine of rotor radius $R$, operating in a free-stream with speed $U_{\infty}$ and turbulence intensity $\mathcal{I}_{\sigma}$. This model relies on the basic assumption that the turbine power coefficient $C_{P}$ is independent of turbulence, instead being only function of the turbine operating regime (tip-speed ratio and Reynolds number). While this might seem a strong assumption, with the authors mentioning this is an upper bound for extracted power, some field studies on real-scale turbines have elaborated on this model obtaining accurate predictions ${ }^{9,10}$, suggesting this assumption is reasonable for large-scale wind turbines operating in the atmospheric boundary layer.

Some studies have however highlighted that free-stream frequency content also affects power harvesting mechanisms: for instance, ref. 17 reports simulations of harmonic gusts on a wind turbine, observing that power harvested from the flow is maximum for gust frequencies between $0.05 \mathrm{~Hz}$ and $0.7 \mathrm{~Hz}$, and decreases rapidly for higher frequency gusts, while lower frequencies do not provide any benefit in terms of generated power; ref. 18 also reports that the inflow frequency content modulates a model tidal turbine power output. Similarly, ref. 19 models the effects of turbulence on harvested power spectrally by means of a transfer function

$$
\phi_{P}(f)=G(f) \phi_{u}(f)
$$

where $\phi_{P}(f)$ and $\phi_{u}(f)$ are the power spectral densities of harvested power and incoming velocity respectively, with $G(f) \simeq 1$ at low frequencies and $G(f) \propto f^{-2}$ in the inertial subrange. As such, this transfer function is heavily skewed towards low-frequency contributions and their study suggests that a wind turbine will harvest more power from a flow where more energy is present as slower fluctuations, or spatially as larger eddies. A similar behaviour is observed in ref. 20 for wind turbines of radically different sizes, ranging from model-scale to full-scale, with diameters in the order of $10^{-1} \mathrm{~m}$ to $10^{2} \mathrm{~m}$, and an analytical approach is presented to explain the presence of the -2 slope in the transfer function; ref. 21 also reports a similar behaviour with a transfer function favouring low-frequency fluctuations, noting how this effect is mediated by the slope of the blade's lift curve near stall.

Lastly, some papers have shown that intermittency in the base flow velocity can also be used to predict torque fluctuations, and thus in principle power fluctuations, of a wind turbine subject to a turbulent inflow; ref. 22 reports that the probability distribution of 
torque increments can be directly related to base flow intermittency, then showing that some synthetic gusts models paired with blade-element method codes can successfully replicate these torque fluctuations. This is even more important when considering that the wake generated by a wind turbine is also highly intermittent ${ }^{23}$, and can affect the power harvesting mechanisms of downstream turbines in wind farms.

In the current study, the performances of a model wind turbine will be characterized, in the mean sense by observing the thrust and power generated by the turbine, statistically by observing the magnitude of power and torque fluctuations, and in Fourier-space by presenting the spectra of thrust fluctuations, when subject to different turbulent base flows. As the reviewed literature has outlined, turbulence intensity alone might not be the single parameter driving changes in the turbine performance figures; nonetheless, this is often the sole turbulence parameter presented in many of the studies reviewed. To ascertain whether this is a valid assumption, it is necessary to separate the effects of different freestream turbulence characteristics on the turbine performance by operating a turbine in flows with similar values of free-stream turbulence intensity and different timescales along with, conversely, flows with comparable timescales coupled with different intensities; this is the main novelty of this paper, which has been made possible by the usage of an active grid to generate bespoke flows. Comparison with similar studies undertaken on aerofoils at similar and larger Reynolds numbers will be carried out to determine whether these observations will hold at larger Reynolds numbers, and thus for real-scale wind turbines, where research on these aspects is scarce.

The paper is structured as follows: section II includes a description of the wind tunnel, the active grid and the model wind turbine used during this study, along with a detailed description of the measurement techniques and their calibration procedures, as well as a detailed presentation of the data reduction techniques that have been used to obtain meaningful data from the experimental measurements; section III reports the results obtained during the measurements, with the effects of turbulence intensity and scale separately analyzed on the power and thrust generated by the model turbine, in conjunction with a discussion on the validity of these results when compared to previous published works in literature; section IV summarises the main findings of this current work.

\section{METHODOLOGY}

\section{A. Facility}

The experiments were carried out in an open-return suction wind tunnel in the University of Southampton. This has a rectangular test section with cross-section of $0.9 \mathrm{~m} \times 0.6 \mathrm{~m}$ and a fetch of $4.5 \mathrm{~m}$.

An active grid similar in design to the one outlined in ref. 24 has been placed at the inlet of the test section to generate turbulent flows. This is composed of an $11 \times 7$ grid of rods, with a mesh spacing of $M=81 \mathrm{~mm}$; a picture of the active grid installed in the wind tunnel test section is reported in fig. 1. A Pitot probe has been placed 2.5 $M$ upstream of the active grid to measure and set the value of $U_{\infty}$ in the wind tunnel; the bulk free-stream velocity has been kept constant to $8 \mathrm{~m} / \mathrm{s}$ by a PID controller implemented in MATLAB for all test cases presented in this work. Two sets of diamond wings similar to the ones employed in ref. 25 have been installed on the grid; the first set was pierced to reduce the maximum grid blockage to $70 \%$, while the second set was completely solid, for a maximum grid blockage of $95 \%$. The active grid has been operated in double-random asynchronous mode as described in ref. 26 , with both the angular velocity of the rods and the time between changes in direction being chosen randomly in a predetermined interval.

Previous research undertaken in this facility using the same active grid have found turbulence intensity to decay with a power law having exponent of 1.25 with distance from the $\operatorname{grid}^{27}$, for different wing geometries and grid routines, in line with studies carried out in similar facilities. ${ }^{28,29}$ 


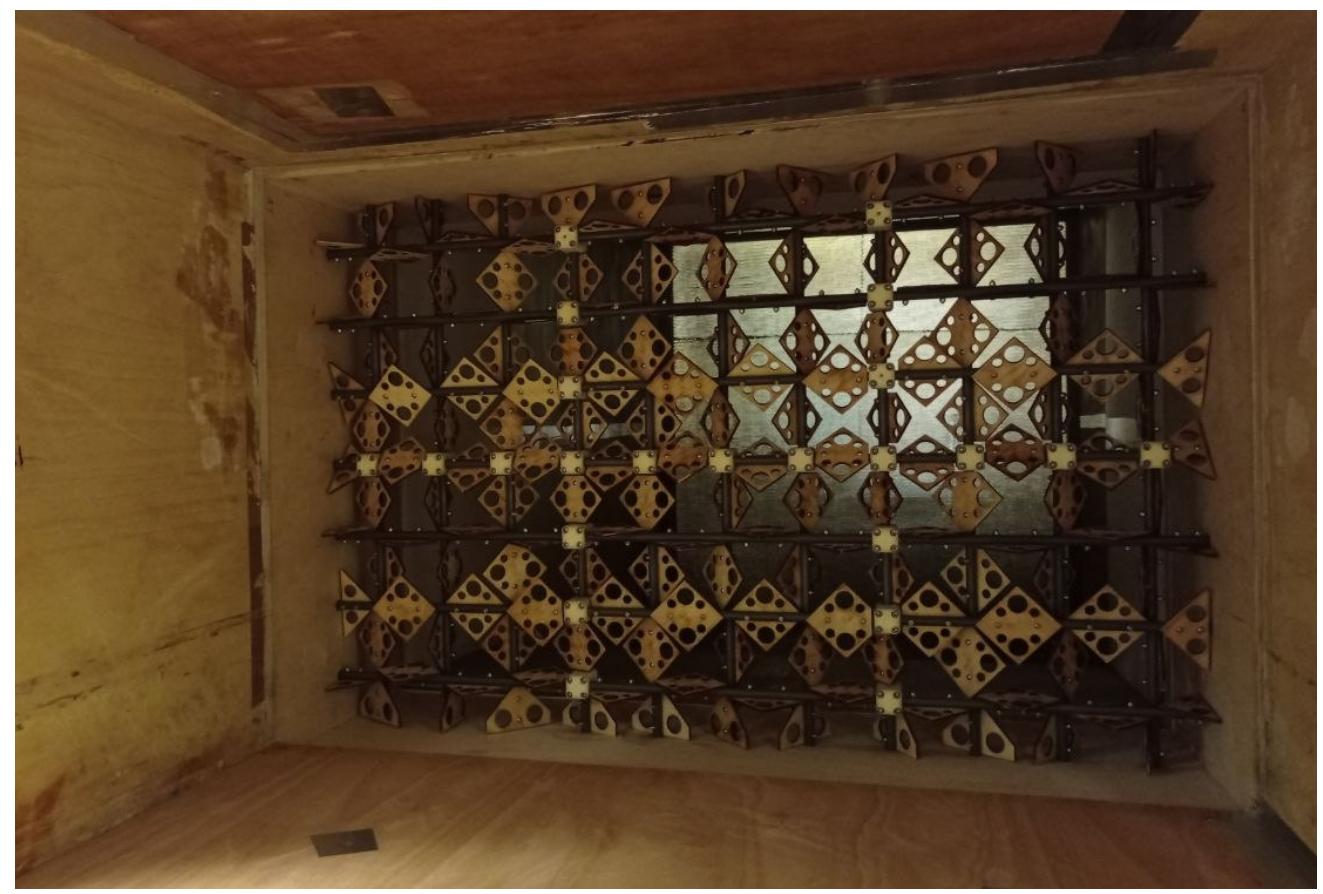

FIG. 1. The active grid in the wind tunnel, seen from downstream, with the low-blockage wings installed

\section{B. Model wind turbine}

The model wind turbine used during these test is a speed-controlled, fixed-pitch three bladed turbine, whose rotor has been connected to a permanent-magnet brushed DC generator. The generator has not been connected to external power sources, with the only source of motion being the aerodynamic torque generated by the turbine rotor. The model turbine rotor has been placed at a streamwise distance of $36 M$ downstream of the active grid, with the rotor hub at the center of the test section. The distance between the rotor plane and the active grid has been chosen as a balance between concerns on the maximum turbulence intensity the model wind turbine would be subject to, decreasing with increasing distance from the active $\operatorname{grid}^{26,29}$, and the necessity of having enough fetch of the test section downstream of the model to allow full wake development not to invalidate the results; this location results in roughly $1.6 \mathrm{~m}$ available for the wake to develop, corresponding to 9 rotor diameters.

The blades installed on the model wind turbine have been 3D-printed in-house with a stereolitography printer; these have a tip-radius of $90 \mathrm{~mm}$, for a rotor diameter of $2 R=0.18 \mathrm{~m}$, resulting in a blockage ratio of $4.7 \%$ in the facility used, computed as the ratio between the rotor swept area and the facility cross-sectional area. A NACA 63-418 aerofoil has been used along the whole blade, with the chord and twist distributions along the blade span reported in fig. 2. The diameter-based Reynolds number of the experiments, based on a mean free-stream velocity of $8 \mathrm{~m} / \mathrm{s}$ is $R e_{D}=9.6 \times 10^{4}$; the chord-based Reynolds number, based on the tip chord and the angular velocity at maximum power generating tip-speed, is $R e_{c}=2.6 \times 10^{4}$.

The turbine speed is measured by means of a Broadcom AS22 incremental optical rotary encoder installed on the turbine shaft, whose output is processed by an Arduino Uno board. This consists of a codewheel having a resolution of 360 counts per shaft revolution, acting as a reflective optical switch; the encoder outputs two square wave trains having 360 periods per physical revolution of the shaft, at a phase angle of 90 electrical degrees from each other. The Arduino board determines the shaft angular velocity by counting the number of rising 


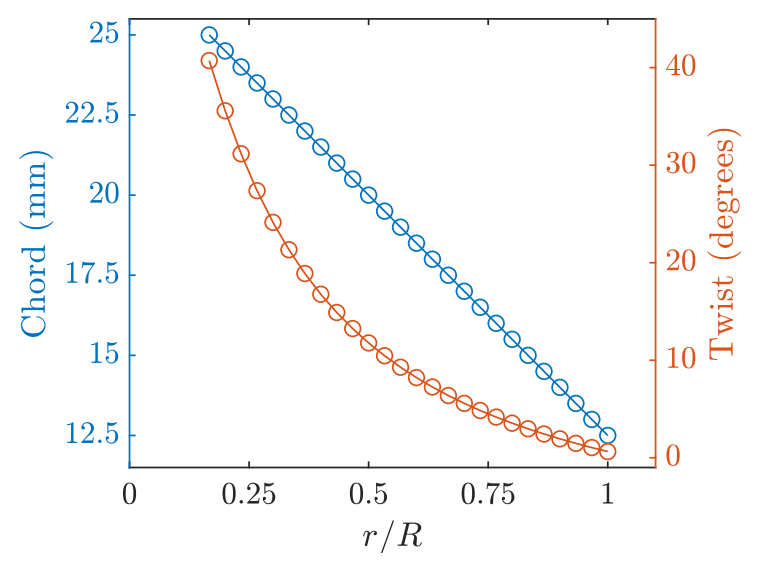

FIG. 2. Chord and twist distributions along the blade span

edges in one of these two signals over a predefined time interval of $250 \mathrm{~ms}$; as the angular position of the shaft is discretised to $1 / 360$ of a revolution, the turbine angular velocity is measured as multiples of $0.67 \mathrm{rev} / \mathrm{min}$. Moreover, as the resulting angular velocity is obtained as a summation of a $250 \mathrm{~ms}$ long signal, fluctuations having a frequency higher than $4 \mathrm{~Hz}$ are filtered out.

The rotor angular velocity is set by means of an H-bridge circuit to which the DC machine is connected, controlled by a $1.6 \mathrm{kHz}$ pulse-width modulation signal generated by the same Arduino board; a PID controller implemented by the Arduino actively sets the turbine angular velocity by controlling the H-bridge MOSFET switches.

The mechanical torque generated by the turbine has been estimated from the current in the DC machine winding; this is an often-used approach for lab-scale wind turbine experiments $^{30,31}$, where the installation of a rotating torque transducer is challenging due to the small dimensions of the turbine. The torque exerted on the DC machine shaft is directly proportional to the winding current $^{32}$; the torque-current relationship has then been modelled as

$$
Q_{s h}=K_{T} I+Q_{f}(\omega)
$$

where $Q_{s h}$ is the mechanical torque to the machine shaft, $I$ is the current in the generator winding, $Q_{f}$ is the torque lost to friction, in this case assumed only function of the rotor angular velocity $\omega$, and $K_{T}$ is a proportionality constant. To measure $K_{T}$, one can take advantage of a property of permanent magnet DC machines for which, under negligible loading,

$$
V=K_{E} \omega-R_{0} I
$$

where $V$ is the voltage drop across the machine poles, $R_{0}$ is the machine internal resistance, and

$$
K_{E}=K_{T}^{-1}
$$

when expressed in appropriate units. Measurement of $K_{E}$ has therefore been carried out by operating the DC machine as a motor for different values of $V$, measuring the machine angular velocity and current draw. Similarly, $Q_{f}(\omega)$ has been estimated by logging the current draw of the machine, disconnected from the turbine rotor, at constant $\omega$. The current in the generator winding has been measured by measuring the voltage drop across a $0.1 \Omega$ shunt resistor, sampled by a 12 bit analog-to-digital converter at a frequency of $4 \mathrm{~Hz}$.

The aerodynamic forces generated by the turbine have been measured with a Mini40 load cell, manufactured by ATI, sensitive to forces and torques along and around all axes. The sensitivity of the instrument to streamwise loads has been estimated by repeatedly loading 
and unloading the load cell with a calibration weight having mass of $0.1 \mathrm{~kg}$, generating a force comparable to the one exerted by the turbine on the load cell; from this procedure, a sensitivity of $0.5 \mathrm{mN}$ has been found. Calibration of this load cell has been carried out by the manufacturer, and no additional calibrations of this device have been undertaken prior to the experiments. Drift of the load cell with respect to time has been corrected for by taking zero-force readings before and after an acquisition, then assuming a linear drift of the balance output with time. The load cell output has been sampled with a dedicated National Instruments USB-6212 data acquisition card at a frequency of $10 \mathrm{kHz}$. The force timeseries have been filtered with a digital Butterworth low-pass filter having a cutoff frequency of $1 \mathrm{kHz}$; additionally, a sixth-order Butterworth band-stop filter between the frequencies of $18 \mathrm{~Hz}$ and $27 \mathrm{~Hz}$ has also been applied to filter out the contributions given by structural vibrations of the turbine mast. Defining the Strouhal number $S t$ as the reduced frequency

$$
S t=\frac{f 2 R}{U_{\infty}}
$$

where $R=0.09 \mathrm{~m}$ is the turbine radius and $U_{\infty}$ is the free-stream speed, this corresponds to a band-stop filter removing spectral contributions between $S t$ of 0.4 and 0.6 . Observing the inflow spectra reported in fig. 6 , these frequencies fall in the inertial subrange for all test cases here reported; as the angular velocities at which the wind turbine operates are always higher than half the filtered frequencies, it can be expected from the data reported in refs. 19 and 20 that this band-stop filter will act in the power-law range of the power spectra.

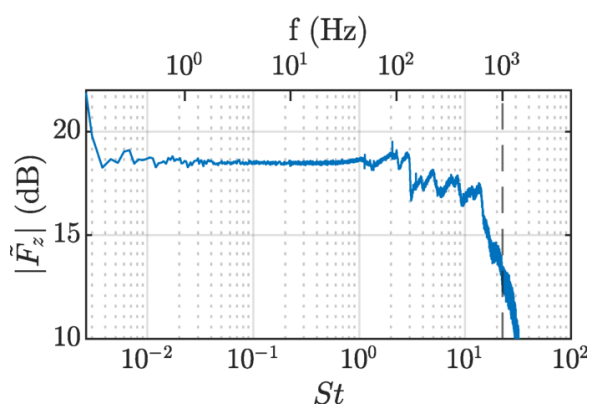

FIG. 3. Frequency response of the ATI Mini40 load cell to an impulse force in the mast direction; the vertical dashed line denotes the cutoff frequency of the Butterworth low-pass filter

The force signals recorded by the load cell will be used to compute the spectra of thrust fluctuations: to determine whether the load cell can adequately measure high-frequency force components, the output of the load cell to an impulse in the vertical direction (parallel to the turbine mast) has been recorded; this impulse has been applied to the load cell by means of a small aluminium rod. The frequency content of the force signal recorded by the load cell is plotted in fig. 3 versus both frequency $f$ and reduced frequency St. The load cell response is flat for frequencies under $130 \mathrm{~Hz}$, or $S t=3$, after which it exhibits a more irregular response settling to a value approximately $1.5 \mathrm{~dB}$ lower. The frequency response then falls considerably for $S t$ larger than 15 , or frequencies of $660 \mathrm{~Hz}$ and higher, lower than the filter cutoff frequency. The frequency content of the thrust measured by the load cell has been presented in fig. 23: no meaningful data is observed for Strouhal numbers larger than 1 and, as the load cell response is mostly flat in this frequency range, no correction to the acquired force signals has been applied.

Prior to the experiments, the power generated by the wind turbine has been estimated with the blade-element method implemented in QBlade ${ }^{33}$. The aerofoil $C_{l}$ and $C_{d}$ values have been estimated with the panel method implemented in $\mathrm{XFOIL}^{34}$, specifying a freestream Reynolds number of $2.4 \times 10^{4}$ and a free-transition coefficient $N_{\text {crit }}$ of 5 ; these have been reported in fig. 4a. The power generated by the model turbine is presented as the 

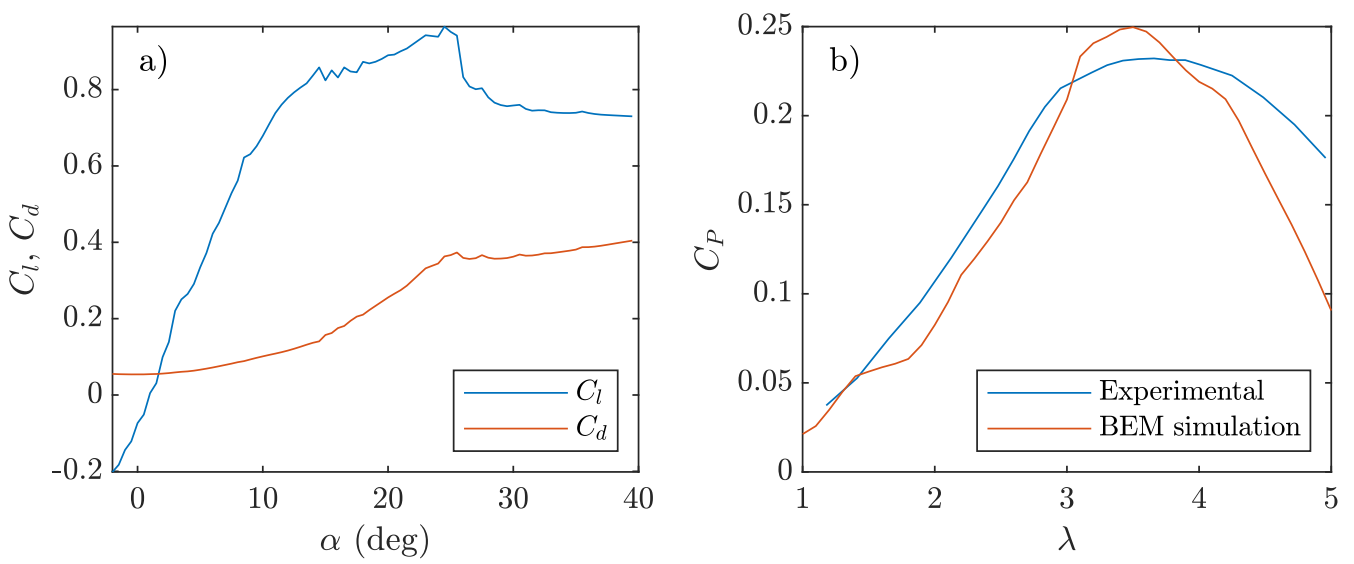

FIG. 4. (left) $C_{l}$ and $C_{d}$ for the NACA $63-418$ aerofoil estimated by XFolL; (right) estimated and actual power coefficient $C_{P}$ versus tip-speed ratio $\lambda$ from BEM simulations and measurements in laminar free-stream

adimensional power coefficient $C_{P}$, defined as

$$
C_{P}=\frac{Q_{\mathrm{sh}} \omega}{\frac{1}{2} \rho U_{\infty}^{3} \pi R^{2}}
$$

where $\omega$ is the turbine angular velocity, $R$ is the turbine rotor radius, $U_{\infty}$ is the free-stream velocity, $\rho$ is the fluid density, $Q_{\mathrm{sh}}$ is the mechanical torque generated as estimated from eq. (3), and the mechanical power harvested by the turbine is estimated as the product of shaft torque and angular velocity; the turbine speed is presented as the tip-speed ratio $\lambda$, defined as

$$
\lambda=\frac{\omega R}{U_{\infty}}
$$

The comparison between the power coefficient predicted by the BEM simulation and the wind tunnel results is reported in fig. 4b. The experimental curve has been obtained in the wind tunnel described in section II A, with the active grid removed, in which a baseline turbulence intensity of $0.3 \%$ is attained. Important differences between the predicted and measured turbine $C_{P}$ are visible, with the BEM code overestimating the peak power generated by the turbine, and underestimating the $C_{P}$ at higher $\lambda$. Nonetheless, this method can still provide a reasonable first approximation for the flow around the turbine blades in laminar free-stream conditions.

To visualize the nature of the flow around the turbine blades, fig. 5 a reports the starting position of the separation bubble on the turbine blade suction side, as a function of the tip-speed ratio $\lambda$ and the adimensional position along the blade span $r / R$, with $R$ being the turbine radius. The local angle of attack is reported in fig. $5 \mathrm{~b}$, with the angles of $15^{\circ}$ and $25^{\circ}$ being highlighted in the plot: these correspond, in the aerofoil polar reported in fig. 4a, to the first value of $\alpha$ for which the $C_{l}(\alpha)$ curve is no longer linear and the value of $\alpha$ that maximises $C_{l}$ respectively. The flow around the turbine blades is expected to be separated for values of $\lambda<3$, and separation is expected to occur uniformly along the blade.

\section{Hot-wire anemometry}

Turbulent characteristics of the base flows generated with the active grid have been measured by means of hot-wire anemometry. Two single-wire probes have been manufactured 

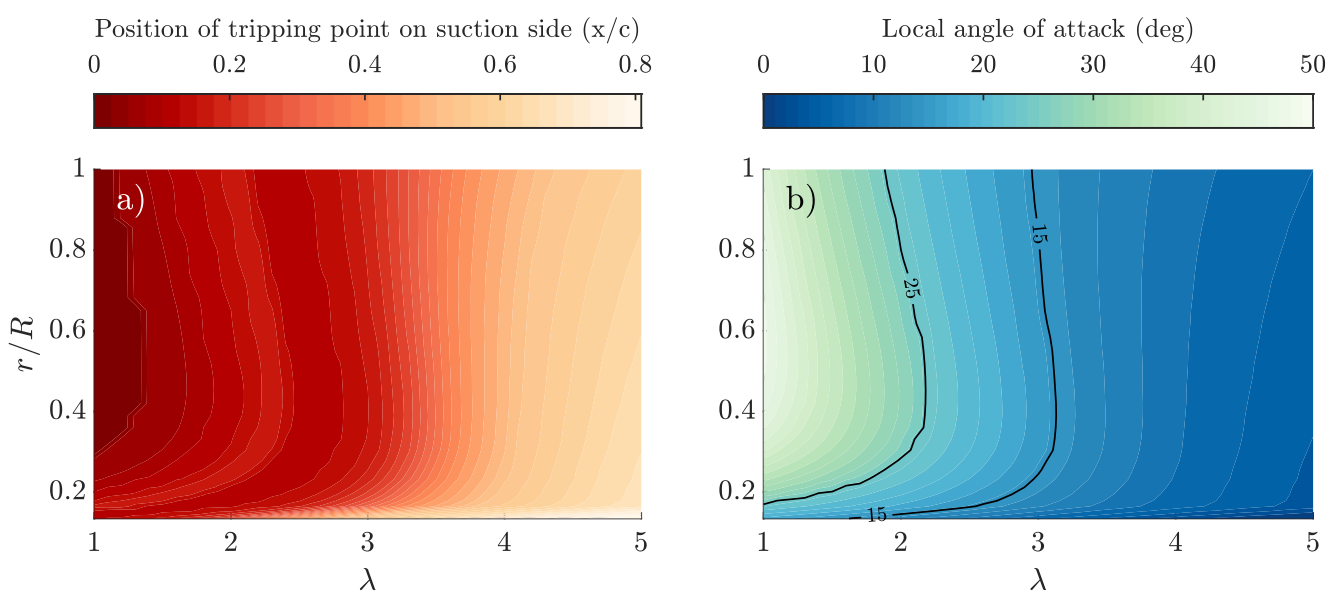

FIG. 5. (left) Position of the tripping point on the suction side of the blades and (right) local angle of attack as a function of tip-speed ratio $\lambda$ and spanwise position on the blade $r / R$

in-house from a $5 \mathrm{\mu m}$ platinum wire with a sensing length of $1 \mathrm{~mm}$, isolated via copper plating of the non-sensing segment of the wire. The probes have been installed on a traverse and moved on a plane $36 M$ downstream of the active grid, at the same streamwise location as the turbine rotor; the measurements have been carried out in an otherwise empty test section.

The properties of the low turbulence base flows obtained with low blockage grid wings have been sampled on a $5 \times 2$ equally spaced grid on a stream-normal plane, spanning vertically from $z / 2 R=-0.55$ to 0.55 , and horizontally from $y / 2 R=-0.28$ to 0.28 ; the higher-turbulence base flows generated by the higher blockage wings have been measured instead on a $3 \times 4$ grid ranging from $z / 2 R=-0.55$ to 0.55 and $y / 2 R=-0.42$ to 0.42 .

The probes have been connected to a constant-temperature anemometer operating at an overheat ratio of 0.8 ; the signal has been conditioned with an analog low-pass filter having a cutoff frequency of $9.6 \mathrm{kHz}$, then sampled at a frequency of $20 \mathrm{kHz}$ with a 16 bit analogto-digital converter integrated in a National Instruments USB-6212 DAQ board. For each position, acquisitions have been carried out for $3 \mathrm{~min}$ to $6 \mathrm{~min}$, to observe convergence of the velocity standard deviation to $\pm 0.5 \%$. This confidence interval has been computed, for each velocity signal acquired, with a bootstrap algorithm as delineated in ref. 35: 100 bootstrap replications of the original velocity signal, each consisting of 5000 samples, have been extracted, and the uncertainty on the obtained velocity standard deviation is obtained from the probability distribution function of the standard deviations of all bootstrap replications.

The probes have been calibrated against a Pitot tube located at the same streamwise distance from the grid. While calibrating, the grid has not been removed from the test section, and its wings have been rotated parallel to the incoming flow to result in the smallest blockage possible; a turbulence intensity of $3 \%$ was measured for this configuration, and its effect on the Pitot probe readings has been neglected. To compensate for the hot-wire drift over time, pre- and post-calibrations have been carried out, with the probes drift assumed linear with time. Additionally, the temperature correction outlined in ref. 36 has been applied to the acquired hot-wire signals.

\section{Data reduction}

The effects of turbulence on the turbine performance characteristics have been investigated by means of a reduced set of parameters, namely the flow turbulence intensity

$$
\mathcal{I}_{\sigma}=\frac{u^{\prime}}{\bar{U}}
$$


where $u^{\prime}=\sqrt{\overline{u^{\prime}(t)^{2}}}$ is the standard deviation of the fluctuating (i.e. zero-mean) streamwise component of velocity $u^{\prime}(t)$, and $\bar{U}$ is the mean streamwise velocity; and the integral time scale

$$
T_{0}=\int_{0}^{\tau_{0}} \rho_{u u}(\tau) d \tau
$$

where $\rho_{u u}(\tau)$ is the autocorrelation factor of $u^{\prime}(t)$ and $\tau_{0}$ is the first zero of $\rho_{u u}(\tau)$. To better estimate the integral time scale for a given velocity timeseries, an ensemble-averaging procedure similar to the one described in ref. 37 has been carried out: initially, the full zeromean velocity signal $u^{\prime}(t)$ has been used to compute a first approximation of $T_{0}$ according to eq. (10); the original velocity signal is then divided in $N_{w}$ windows, each having a duration of 200 times the initial $T_{0}$ approximation, with a different autocorrelation factor $\rho_{u u}(\tau)$ being computed for each window; these $N_{w}$ distinct autocorrelation factors are then averaged to obtain an ensemble-averaged $\bar{\rho}_{u u}(\tau)$ which is then used in eq. (10) to obtain the final estimate of $T_{0}$.

To relate the flow integral time scale to the turbine properties, this last quantity is normalised by the rotation period of the wind turbine at peak power harvesting regime $T_{\mathrm{rev}}=20 \mathrm{~ms}$

$$
\mathcal{T}_{0}=\frac{T_{0}}{T_{\text {rev }}}
$$

Physically, this is a measure of how many rotations the turbine completes while being traversed by a single large scale structure. It is possible to relate this quantity to the more commonly used ratios between integral length scale $L_{0}$ and turbine diameter $2 R$ or turbine tip-chord $c_{\text {tip }}$ by means of Taylor's frozen turbulence hypothesis

$$
\begin{gathered}
\frac{L_{0}}{2 R}=\frac{T_{0} U_{\infty}}{2 R}=\frac{U_{\infty} T_{\text {rev }}}{2 R} \mathcal{T}_{0} \\
\frac{L_{0}}{c_{\text {tip }}}=\frac{T_{0} U_{\infty}}{2 R}=\frac{U_{\infty} T_{\text {rev }}}{c_{\text {tip }}} \mathcal{T}_{0}
\end{gathered}
$$

where $U_{\infty}=8 \mathrm{~m} / \mathrm{s}, R=90 \mathrm{~mm}$ and $c_{\text {tip }}=15 \mathrm{~mm}$ for this study.

Both $\mathcal{I}_{\sigma}$ and $\mathcal{T}_{0}$ are computed from the instantaneous velocity signals obtained via hotwire anemometry, as described in section II C: these are single-wire probes aligned with the horizontal direction, and thus measure the instantaneous velocity in the streamwise-vertical plane, perpendicular to the wire, including the zero-mean vertical velocity component. Using the fluctuating component of this signal to estimate $\mathcal{I}_{\sigma}$ and $\mathcal{T}_{0}$ instead of $u^{\prime}(t)$ in eqs. (9) and (10) does in principle lead to an error in the estimation of those quantities; however, given the mean flow is oriented in the direction of $u^{\prime}$, we have neglected the effect of this on the computed turbulence parameters.

The flows generated by the active grid are not characterized by a uniform distribution of turbulence intensity and integral time scale on the turbine swept area; for this reason, the values of these parameters as presented in section III are obtained by a disc-averaging process conceptually identical to the definition of rotor-equivalent velocity carried out in ref. 9. For no test case, the maximum deviation of turbulence intensity from the mean has exceeded $3.5 \%$ of the mean, while integral time scale has never deviated more than $10 \%$ from the rotor-averaged values.

In addition to the power coefficient $C_{P}$, defined in eq. (7), the model turbine performance will be analyzed in terms of its torque and thrust coefficients (respectively $C_{Q}$ and $C_{T}$ ), considered functions of the tip-speed ratio $\lambda$ defined in eq. (8). These are defined as

$$
\begin{aligned}
C_{T} & =\frac{T}{\frac{1}{2} \rho U_{\infty}^{2} \pi R^{2}} \\
C_{Q} & =\frac{Q_{\mathrm{sh}}}{\frac{1}{2} \rho U_{\infty}^{2} \pi R^{3}}=\frac{C_{P}}{\lambda}
\end{aligned}
$$


where $\omega$ is the turbine angular velocity, $R$ is the turbine rotor radius, $U_{\infty}$ is the average free-stream velocity during the tests, $\rho$ is the fluid density, $T$ is the thrust generated by the turbine, and $Q_{\mathrm{sh}}$ is the mechanical torque generated as estimated from eq. (3). The thrust figures measured by the load cell contain both the contributions of the turbine and the drag generated by the supporting structure (nacelle and mast). To estimate the fraction of drag generated by the turbine rotating parts, the forces acting on the support structure have been removed from the acquired forces with the turbine rotor in place. These last ones have been measured by mounting a dummy turbine hub without blades on the motor shaft, with measurements on the forces generated in this configuration measured for all base flows the turbine has been subject to.

In addition, to quantify the unsteady mechanical load to the generator, we define, given a torque timeseries $Q_{\mathrm{sh}}(t)$, the unsteady torque coefficient

$$
C_{Q}^{\prime}=\frac{Q^{\prime}}{\frac{1}{2} \rho U_{\infty}^{2} \pi R^{3}}
$$

where $Q^{\prime}=\sqrt{\overline{Q_{\mathrm{sh}}^{\prime}(t)^{2}}}$ is the standard deviation of the fluctuating torque timeseries $Q_{\mathrm{sh}}^{\prime}(t)$. Similarly, we define the unsteady power coefficient as

$$
C_{P}^{\prime}=\frac{\overline{Q_{\mathrm{sh}}^{\prime}(t) \omega^{\prime}(t)}}{\frac{1}{2} \rho U_{\infty}^{3} \pi R^{2}}
$$

where $\omega^{\prime}(t)$ is the fluctuating component of angular velocity for a given timeseries. Note that the prime symbol in $C_{P}^{\prime}$ does not refer to the fluctuating (zero-mean) component of the $C_{P}(t)$ generated by the wind turbine, but rather to the component of time-averaged power coefficient $C_{P}$ which is generated by the coupled fluctuations in the turbine torque and angular velocity. As such, $C_{P}^{\prime}$ is not a function of time, and its value is different from zero.

For each base flow, the turbine has operated in a range of angular velocities $\omega$ between $1000 \mathrm{rev} / \mathrm{min}$ and $4200 \mathrm{rev} / \mathrm{min}$, corresponding to a range of reduced speeds $\lambda$ from 1 to 5 ; multiple acquisitions of the performance coefficients have been carried out to reduce the uncertainty on the measurements.

The plots presented in section III report the $95 \%$ confidence intervals on the mean values of all performance coefficients as a function of $\lambda$. The uncertainty $\varepsilon_{X}$ on the generic measured coefficient $C_{X}$ is estimated as

$$
\varepsilon_{X}=\sqrt{\left(2 \frac{\operatorname{std}\left(x_{1,1} \ldots x_{1, N}, x_{2,1} \ldots x_{M, N}\right)}{\sqrt{N_{\text {ind }}}}\right)^{2}+\varepsilon_{\text {instr }}^{2}}
$$

where $x_{j, k}$ refers to the $k$-th sample in the $j$-th acquired timeseries of $C_{X}(\lambda), \operatorname{std}()$ refers to the standard deviation of the set in parentheses, $\varepsilon_{\text {instr }}$ is the uncertainty introduced by the measuring instrument, and $N_{i n d}$ refers to the number of statistically independent samples in the dataset analyzed.

To estimate $N_{i n d}$, two instantaneous measurements $x_{i, k}$ and $x_{j, k}$ are considered to be statistically independent if these are separated in time by more than $\delta t$

$$
\delta t=\max \left(\frac{2 R}{U_{\infty}}, 2 T_{0}\right)
$$

with $T_{0}$ being the free-stream integral time scale of the particular base flow the turbine is subject to.

For measurements of mechanical power and torque, four distinct time series of angular velocity and torque at each operating point of the turbine have been acquired in base flows having turbulence intensity lower than $10 \%$, while six time series have been recorded in higher-turbulence conditions. The thrust measurements have instead been acquired once 
for the data reported in figs. 20 to 22, with an additional, longer acquisition being dedicated to the data presented in fig. 23. This was made necessary by the higher standard deviation of the torque signal, requiring a larger number of statistically independent samples to bring the confidence intervals down to an acceptable level, whereas the uncertainty on the mean thrust values is mostly dominated by $\varepsilon_{\text {instr }}$ as standard deviation of these is small.

\section{RESULTS AND DISCUSSION}

\section{A. Base flows}

The model turbine performance has been measured in 11 base flows, with their main characteristics reported in table I. For convenience, the base flows are named with a number and a letter, respectively increasing with integral time scale and turbulence intensity; base flows with the same number thus have comparable values of $\mathcal{T}_{0}$, while test cases with the same letter have approximately the same $\mathcal{I}_{\sigma}$. The case named $1 \mathrm{~A}$ has been used as lowturbulence reference, and obtained with the same procedure described in section IIC for hot-wire calibration. The active grid routine is represented by its nominal Rossby number Ro, defined as

$$
R o=\frac{U_{\infty}}{\bar{\Omega} M}
$$

where $\bar{\Omega}$ is the mean angular velocity of the rods, and $M$ is the grid mesh spacing.

TABLE I. Base flow characteristics

\begin{tabular}{|c|c|c|c|c|c|c|c|}
\hline Name & Grid wings & Ro & $\mathcal{I}_{\sigma}(\%)$ & $\mathcal{T}_{0}$ & $L_{0} / 2 R$ & $L_{0} / c_{\text {tip }}$ & $\overline{\text { Legend }}$ \\
\hline$\overline{1 \mathrm{~A}}$ & - & - & 3.0 & 0.16 & 0.14 & 1.71 & $\overline{0}$ \\
\hline $2 \mathrm{~B}$ & \multirow{6}{*}{ Low blockage } & 2 & 7.5 & 1.05 & 0.93 & 11.20 & $\triangleleft$ \\
\hline $2 \mathrm{C}$ & & 5 & 8.8 & 1.13 & 1.00 & 12.05 & $\triangleright$ \\
\hline $3 \mathrm{D}$ & & 10 & 9.6 & 1.56 & 1.39 & 16.64 & $\square$ \\
\hline $4 \mathrm{E}$ & & 20 & 10.7 & 3.00 & 2.67 & 32.00 & 柁 \\
\hline $5 \mathrm{~F}$ & & 40 & 11.3 & 7.05 & 6.27 & 75.20 & $\star$ \\
\hline $6 \mathrm{~F}$ & & 60 & 11.6 & 11.75 & 10.44 & 125.33 & $\triangle$ \\
\hline $2 \mathrm{~F}$ & \multirow{4}{*}{ High blockage } & 2 & 11.5 & 1.16 & 1.03 & 12.37 & $\frac{\bar{\nabla}}{\nabla}$ \\
\hline $2 \mathrm{G}$ & & 5 & 13.2 & 1.14 & 1.01 & 12.16 & $\diamond$ \\
\hline $3 \mathrm{H}$ & & 12 & 14.8 & 1.59 & 1.41 & 16.96 & $\otimes$ \\
\hline $4 \mathrm{I}$ & & 25 & 16.2 & 3.19 & 2.84 & 34.03 & $凶$ \\
\hline
\end{tabular}

To better appreciate the difference between these conditions, fig. 6 reports the spectra of two families of incoming flows: base flows at low turbulence scale $(2 \mathrm{~B}, 2 \mathrm{C}, 2 \mathrm{~F}$, and $2 \mathrm{G}$ ) $\mathcal{T}_{0}$ have been plotted in fig. $6 \mathrm{a}$, while test cases at constant $\mathcal{I}_{\sigma}(2 \mathrm{~F}, 5 \mathrm{~F}$, and $6 \mathrm{~F})$ have been reported in fig. 6b. From the first figure, it is evident that the flows generated by the active grid at low Ro exhibit, at least in the spectral domain, the same characteristics of canonical passive-grid generated turbulence, with a flat spectrum in the energy containing subrange and an approximately $-5 / 3$ slope in the inertial region; the main difference between these curves is a uniform shift towards more energetic eddies present at all frequencies, compatible with a different turbulence intensity. The flows generated by the active grid operating at $R o=5$ also present a small peak in their spectra for a range of frequencies close to the angular velocities of the active grid rods; this contribution is not present for test cases $2 \mathrm{~B}$ and $2 \mathrm{~F}$, where the active grid has operated at a Rossby number of 2 .

The power spectral densities of the three flows sharing the same $\mathcal{I}_{\sigma}(2 \mathrm{~F}, 5 \mathrm{~F}$, and $6 \mathrm{~F})$ are, on the contrary, quite different: a peak at low frequencies, corresponding to the range of frequencies at which the active grid has operated, is present for the two largest scale flows, and absent in test case $2 \mathrm{~F}$. This results in a different spectral distribution of power 

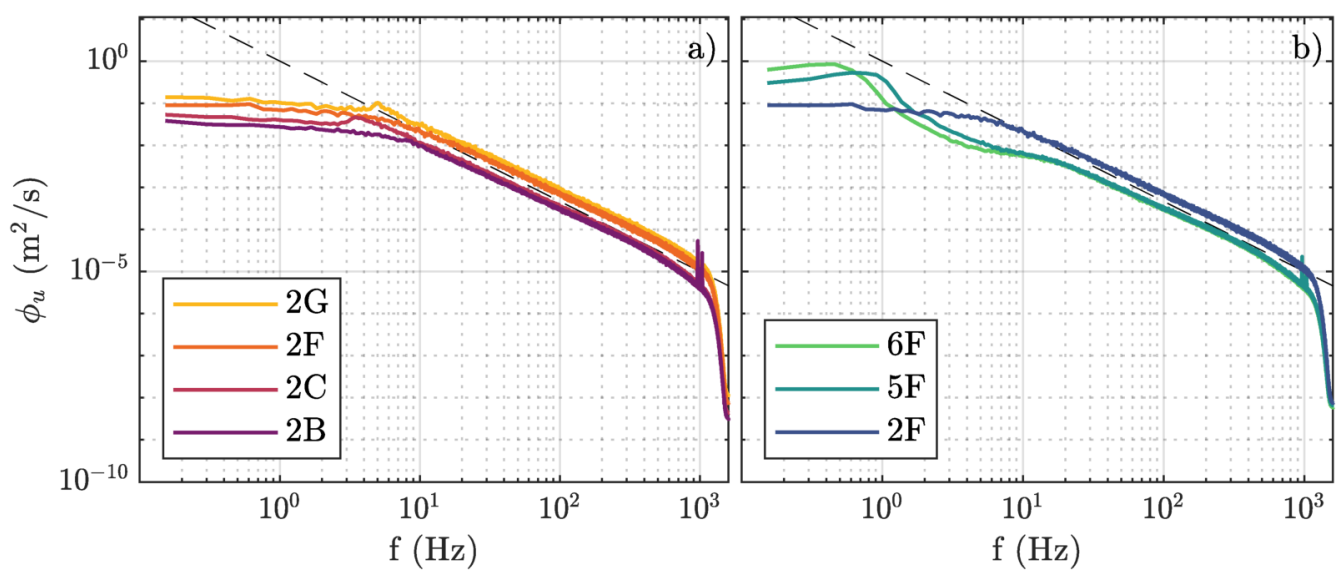

FIG. 6. Spectra of constant $\mathcal{T}_{0}$, increasing $\mathcal{I}_{\sigma}$ flows (left) and constant $\mathcal{I}_{\sigma}$, increasing $\mathcal{T}_{0}$ flows (right) with reference $-5 / 3$ slope (dashed line)

between these cases, with flows at high scale carrying significantly more energy as lowfrequency fluctuations, while being markedly less energetic in the inertial subrange. As their turbulence intensity is similar, these three flows are extremely well suited to verify the validity of the analyses presented by previous literature, such as the models derived from ref. 8 , where $\mathcal{I}_{\sigma}$ is assumed to be the only parameter driving mean power variations regardless of the turbulence frequency content, or the low-pass turbine behaviour delineated in refs. $19-21$.

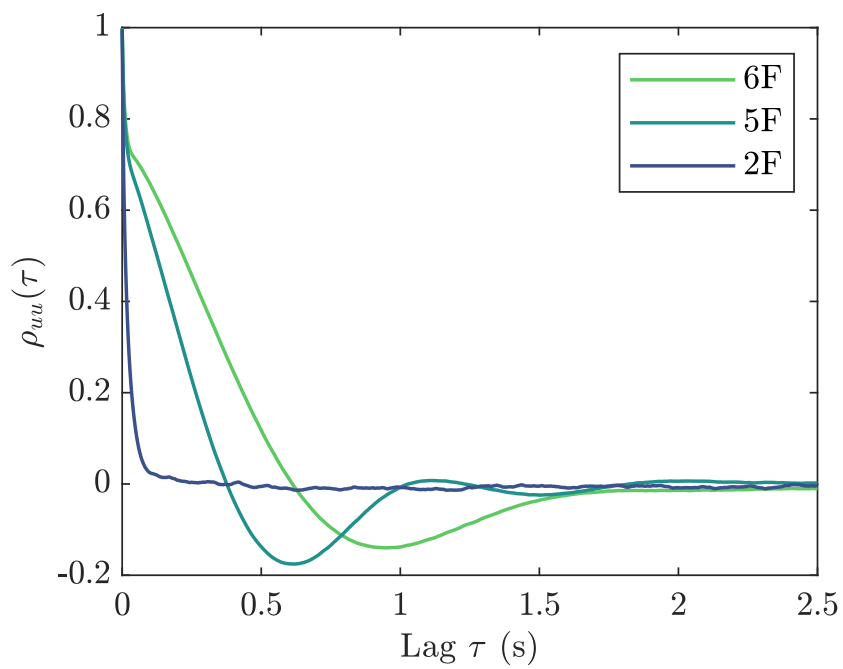

FIG. 7. Autocorrelation coefficient $\rho_{u u}(\tau)$ for the constant $\mathcal{I}_{\sigma}$ flows $2 \mathrm{~F}, 5 \mathrm{~F}$ and $6 \mathrm{~F}$ as a function of lag $\tau$

The autocorrelation coefficients for these last three flows are also reported in fig. 7: flow $2 \mathrm{~F}$ is seen to behave like a classical grid-generated turbulent flow, rapidly losing correlation with itself; in comparison, flows $5 \mathrm{~F}$ and $6 \mathrm{~F}$ have a more delayed zero-crossing and afterwards show negative values of $\rho_{u u}(\tau)$. The negative value of the autocorrelation coefficient shows that, for small lags, the fluctuating velocity signal $u^{\prime}(t)$ is similar to its shifted negative $-u^{\prime}(t+\tau)$; this suggests that these two flows can be seen as quasi-periodic gusts superimposed to a turbulent base flow. 


\section{B. Mechanical power and torque}

The power generated by the wind turbine has been measured for all base flows reported in table I, and for $\lambda$ ranging from 1 to 5 . The power curves on the $C_{P}-\lambda$ space are self-similar between different base flows, as reported in fig. 8 with the parameters used to normalize these curves being the maximum power coefficient attained by the model turbine and the tip-speed ratio at which highest mechanical torque is generated $\lambda_{\text {peak }}$; this last parameter was chosen as opposed to the value of $\lambda$ that maximises $C_{P}$ because the torque curves are characterized by a clearer peak, as it will be seen in fig. 11, as opposed to the large range of $\lambda$ for which a constant $C_{P}$ is attained. Being these curves self-similar, their difference can be analyzed by observing how peak $C_{P}$ varies between base flows.

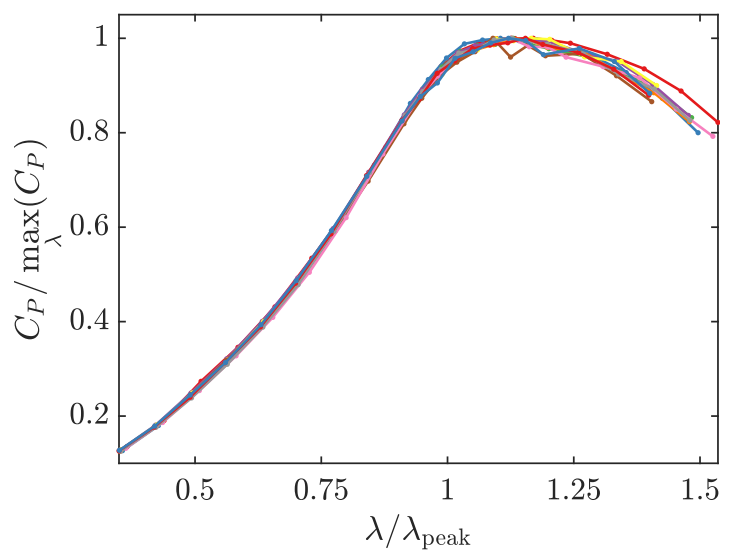

FIG. 8. Self-similarity of power curves $C_{P}(\lambda)$

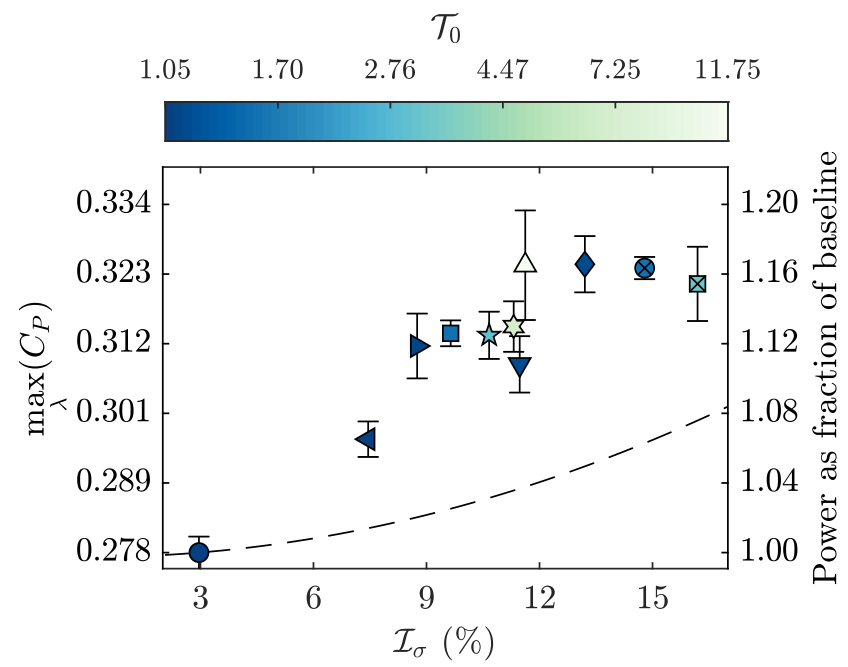

FIG. 9. Dependence of maximum power generated on turbulence characteristics, and (dashed line) parabolic increase in power from ref. 8; note the logarithmic scale of the colour bar. Markers indicate the base flow as reported in the relevant column of table I.

These peaks have been reported in fig. 9 as functions of turbulence intensity $\mathcal{I}_{\sigma}$ and scale $\mathcal{T}_{0}$. From this data, a trend of increasing generated power with turbulence is evident, with both $\mathcal{I}_{\sigma}$ and $\mathcal{T}_{0}$ proving beneficial from the point of view of harvested power. However, the expected parabolic trend with turbulence intensity presented in ref. 8, which is usually assumed to hold true for real scale wind turbines, is not present. In fact, for high values of 
turbulence, the increase in power observed from the data presented is almost double what would be predicted by the model - compare, for instance, the $16 \%$ increase in power yield at highest turbulence intensity versus the $8 \%$ predicted by the analytical model. Moreover, the power extracted by the turbine from different base flows at highest $\mathcal{I}_{\sigma}$ is constant, while a large difference could be expected if the parabolic trend were to hold true.
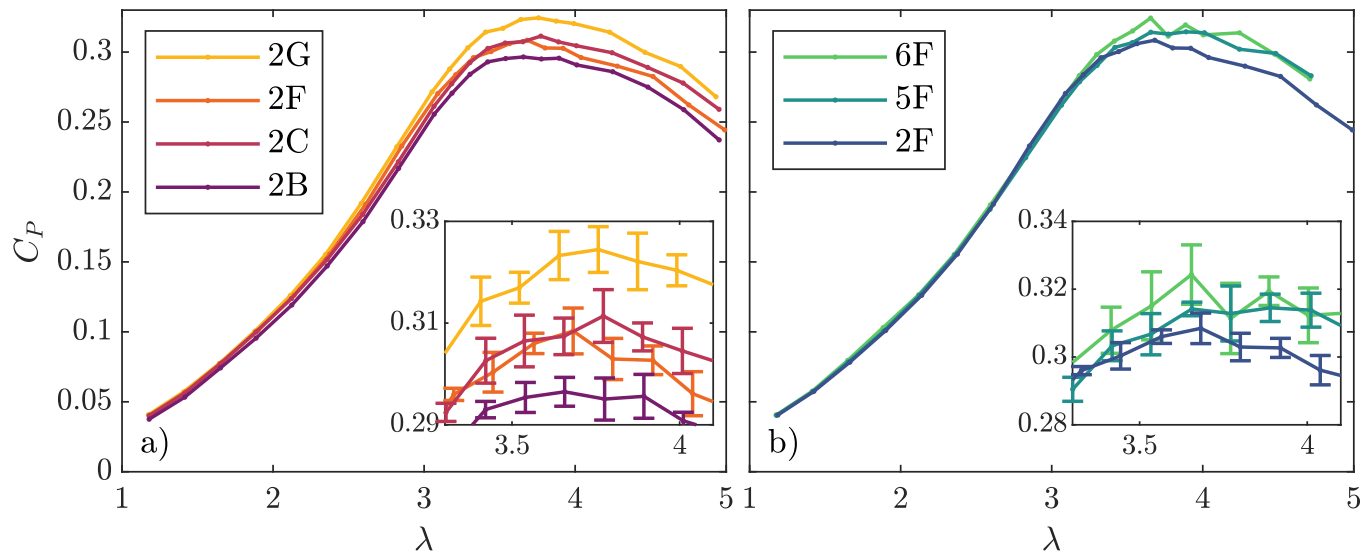

FIG. 10. Power curves for constant $\mathcal{T}_{0} \simeq 1.1$ (left) and constant $\mathcal{I}_{\sigma} \simeq 11.5 \%$ (right)

The effect of turbulence intensity alone can be isolated by analysing the power trends for base flows $2 \mathrm{~B}, 2 \mathrm{C}, 2 \mathrm{~F}$ and $2 \mathrm{G}$, which share a close value of $\mathcal{T}_{0} \simeq 1.1$ and a large range of $\mathcal{I}_{\sigma}$ from $7.5 \%$ to $13.2 \%$; the relative power curves have been reported in fig. 10a. The effect of turbulence intensity alone is that of a clear increase in the turbine power yield, with the curves obtained for flows $2 \mathrm{~B}, 2 \mathrm{~F}$ and $2 \mathrm{G}$ distinctively staggered along the vertical axis, for all values of $\lambda$; some overlap between the two intermediate flows $2 \mathrm{C}$ and $2 \mathrm{~F}$ is however present, despite the important difference in turbulence intensity between these base flows.

Likewise, the effect of turbulence scale can be observed from the power curves obtained with flows $2 \mathrm{~F}, 5 \mathrm{~F}$ and $6 \mathrm{~F}$, sharing a close value of $\mathcal{I}_{\sigma}$, reported in fig. $10 \mathrm{~b}$. Despite the larger uncertainty in the measurements for base flows $5 \mathrm{~F}$ and $6 \mathrm{~F}$, a trend of increasing power can be clearly observed connected with larger flow scales, with flow $2 \mathrm{~F}$ clearly resulting in less power extracted by the turbine despite the same turbulence intensity. Unlike the power curves obtained in different $\mathcal{I}_{\sigma}$, negligible differences in power are observed for low values of $\lambda$, with the turbulence scale affecting the power curves only at high values of tip-speed ratio.

To more easily tackle the mechanisms that lead to power variations, it is advantageous to independently study the effects of turbulence on the individual parameters on which power depends. Given a time window with a timeseries of torque and angular velocity, the mean power coefficient is

$$
C_{P}=\frac{\overline{Q(t) \omega(t)}}{\frac{1}{2} \rho U_{\infty}^{3} \pi R^{2}}=\frac{\overline{\left(\bar{Q}+Q^{\prime}\right)\left(\bar{\omega}+\omega^{\prime}\right)}}{\frac{1}{2} \rho U_{\infty}^{3} \pi R^{2}}=\frac{\bar{Q} \bar{\omega}}{\frac{1}{2} \rho U_{\infty}^{3} \pi R^{2}}+C_{P}^{\prime}
$$

where $C_{P}^{\prime}$ refers to the fraction of power generated by the combined fluctuations in torque and angular velocity, as defined in eq. (17). As such, under constant $U_{\infty}$, the mean wind turbine power output is increased either with an increase in the torque generated at the same angular velocity, or with greater angular velocity at which the same torque is attained. In addition, a less evident component of power is connected to the coupled fluctuations of torque and angular velocity, with this last contribution not necessarily positive, depending on the combined fluctuations signs.

The torque curve obtained for flow $4 \mathrm{E}$ is reported in fig. 11 as representative of torque curves for all base flows. The curve exhibits a clear peak and is otherwise linear, with the peak closely related to the stall angle of attack of the blades, being the tip-speed ratio 


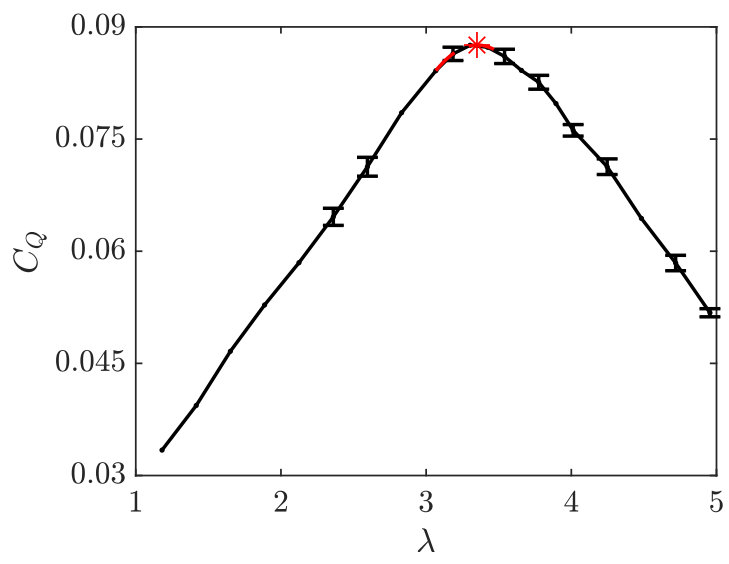

FIG. 11. Torque curve $C_{Q}(\lambda)$ for base flow $4 \mathrm{E}\left(\mathcal{I}_{\sigma}=10.7 \%, \mathcal{T}_{0}=3.00\right)$, and $($ red $)$ estimation of peak torque position; $95 \%$ confidence intervals on power omitted where smaller than $1 \%$ of the measurement

loosely inversely proportional to the blades' average angle of attack. The flow around the blades is attached for $\lambda>\lambda_{\text {peak }}$ and otherwise separated, with the slope of the $C_{Q}$ curve at high $\lambda$ then connected to the $C_{l, \alpha}$ of the aerofoils. This is compatible with the BEM simulation results reported in fig. 5 , which predicted the turbine blades to stall at a $\lambda$ of 3 .

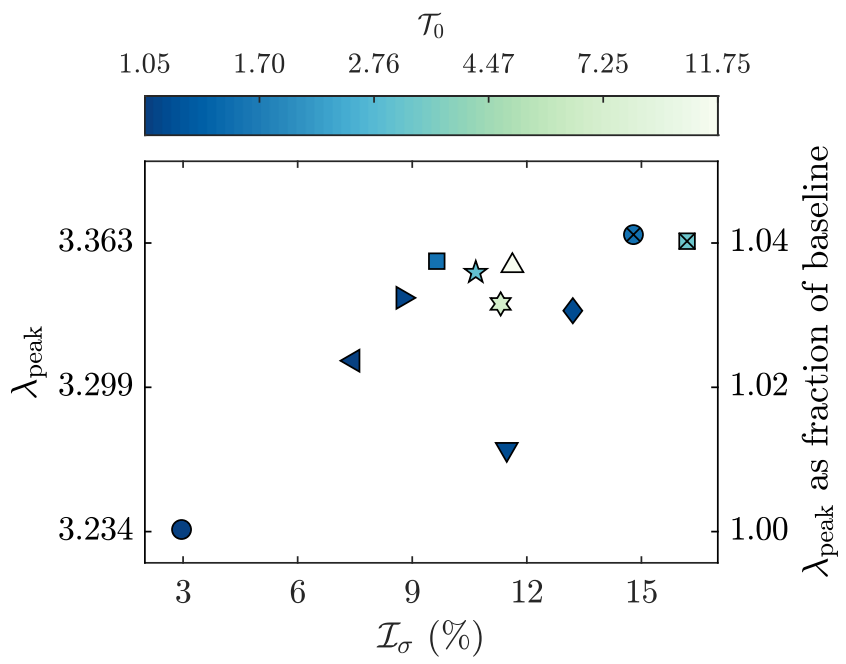

FIG. 12. Dependence of $\lambda_{\text {peak }}$ on the turbulence characteristics

The position of $\lambda_{\text {peak }}$ as a function of the base flow properties is reported in fig. 12, from which it is evident that this aspect can only account for a fraction of power variations previously observed, since the increase in $\lambda_{\text {peak }}$ is always smaller than $4 \%$. Note that to improve the estimation of $\lambda_{\text {peak }}$, the torque curves have been interpolated with a secondorder polynomial with a five-point stencil around the curve maximum, as reported in fig. 11.

Due to the small magnitudes of these changes, finding a definite trend of this parameter with free-stream turbulence properties is not straightforward: the data suggests a simple dependence of $\lambda_{\text {peak }}$ on $\mathcal{I}_{\sigma}$, increasing linearly until a constant value is reached at turbulence intensities greater than $9 \%$, with flow $2 \mathrm{~B}\left(\mathcal{I}_{\sigma}=11.5 \%, \mathcal{T}_{0}=1.16\right)$ being an outlier for this trend. Alternatively, this trend can be interpreted mostly as an increasing function of $\mathcal{T}_{0}$, with constant $\lambda_{\text {peak }}$ attained for $\mathcal{T}_{0}>3$, which would explain the lower value observed for flow $2 \mathrm{~F}$. While this might be tentatively connected to an earlier onset of stall, this 
is not found in literature, with stall angle of attack mostly unaffected, if not delayed, by free-stream turbulence ${ }^{38,39}$.

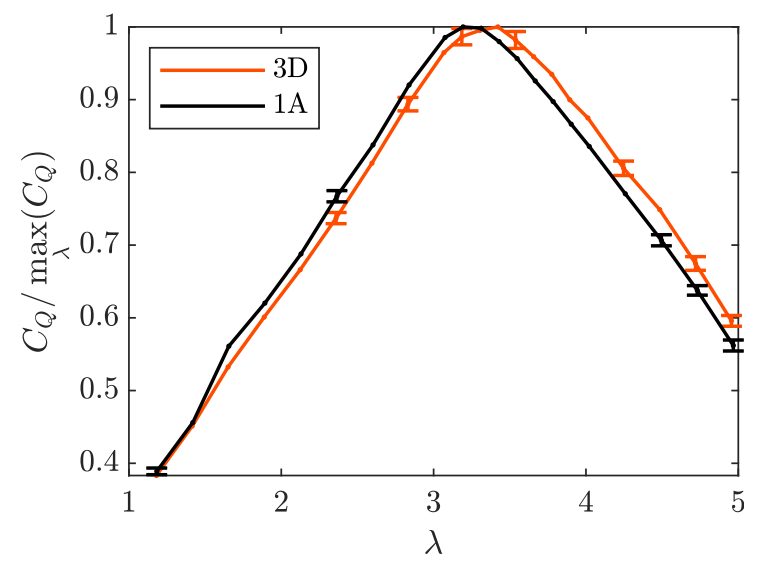

FIG. 13. Torque generated by base flows $1 \mathrm{~A}$ and $3 \mathrm{D}$, normalised to their maximum; $95 \%$ confidence intervals omitted where smaller than $1 \%$ of the measurement

From the point of view of power production, this leads to the result of an increased $C_{P}$ due to the higher value of $\lambda$ at which this peak is attained. To better visualize this aspect, fig. 13 reports the torque curves obtained for the reference base flow and a moderately turbulent test case; to isolate the tip-speed ratio shift of torque, the curves are normalised to their maximum. It appears that the shift in $\lambda_{\text {peak }}$ is representative of a uniform shift of the torque curve towards higher values of $\lambda$, which results in a higher power harvested by the turbine due to the increased angular velocity at which the shaft torque is exerted. While this aspect can explain the initial increase of power for low values of $\mathcal{I}_{\sigma}$, its magnitude is limited, with the increase in $\lambda_{\text {peak }}$ being always lower than $4 \%$ of the baseline value, and thus cannot account for the totality of $C_{P}$ variations observed in fig. 9. Furthermore, with $\lambda_{\text {peak }}$ being constant for high turbulence intensities, this phenomenon cannot result in the variations in power observed for $\mathcal{I}_{\sigma}>10 \%$.

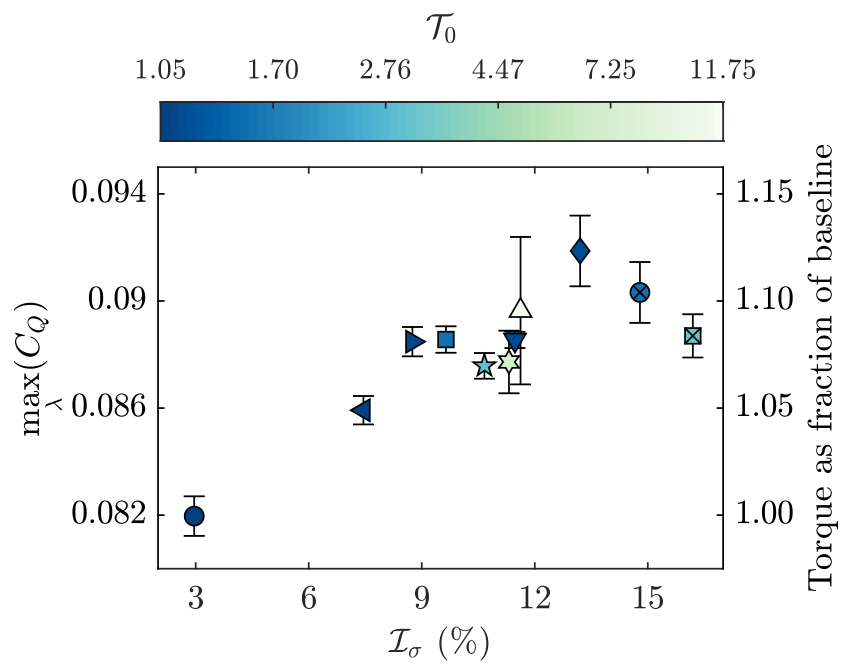

FIG. 14. Dependence of maximum torque on the base flow properties

The maximum torque attained by the turbine also clearly affects the power harvested by the model turbine, with fig. 14 reporting the trend of maximum turbine torque as a function of the turbulence characteristics. Similar to what was observed for maximum power, the 
torque clearly increases with turbulence intensity and the asymptotic trend observed for $\lambda_{\text {peak }}$ is not present, thus explaining the differences in power obtained for large values of $\mathcal{I}_{\sigma}$. Contrarily to what observed for power, peak torque does not appear to be affected by $\mathcal{T}_{0}$, with flows $2 \mathrm{~F}, 5 \mathrm{~F}$ and $6 \mathrm{~F}$ resulting in similar maximum $C_{Q}$.

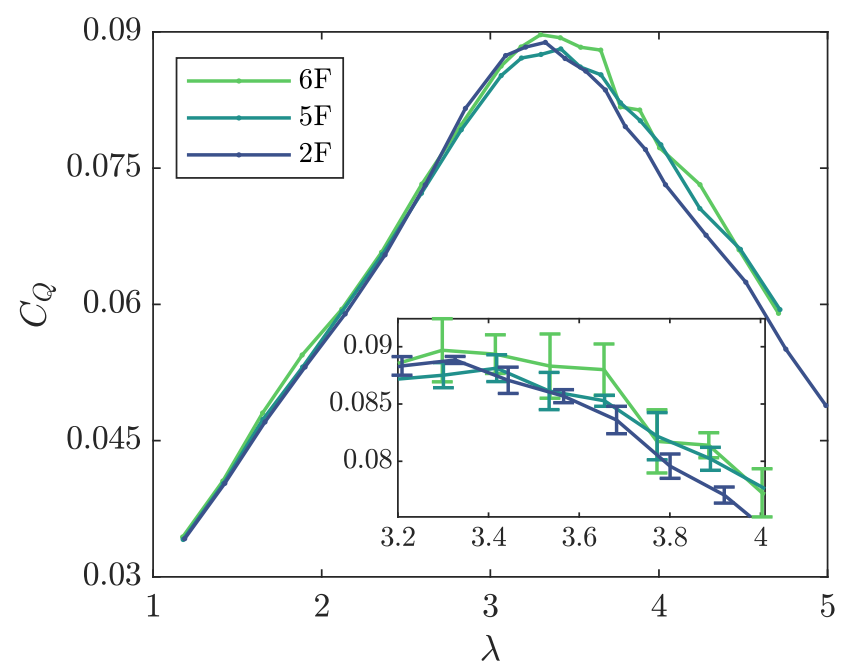

FIG. 15. Torque coefficient attained for base flows $2 \mathrm{~F}, 5 \mathrm{~F}$ and $6 \mathrm{~F}\left(\mathcal{I}_{\sigma} \simeq 11.5 \%\right)$

To explain the effect of $\mathcal{T}_{0}$ on power, the peak torque might thus not be a parameter representative of the full torque curve, and the full curves have to be compared. From the curves reported in fig. 15 it can be appreciated that the main difference caused by the base flows at constant $\mathcal{I}_{\sigma}$ resides in the torque generated close to the blade stall, with the higher $\mathcal{T}_{0}$ flows resulting in a less steep torque curve and a more gentle stall, with high torques being kept for higher $\lambda$, thus leading to an increase in power akin to the one highlighted by the data in fig. 13.

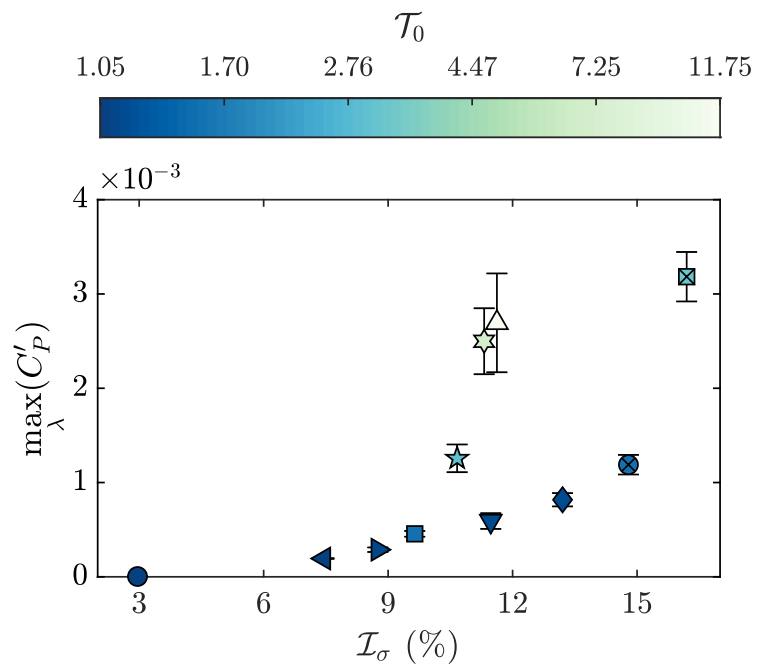

FIG. 16. Dependence of power due to torque and angular velocity fluctuations on the turbulence characteristics

The last contribution to power comes from the coupled fluctuations in torque and angular velocity contained in the term $C_{P}^{\prime}$. This parameter is predominantly affected by free-stream turbulence scale, although a smaller, linear dependency on $\mathcal{I}_{\sigma}$ is also found for base flows 
with $\mathcal{T}_{0}<2$; for no test cases this component of power amounts to more than $1 \%$ of the maximum power generated by the model turbine, thus suggesting this contribution can be neglected with respect to the total model turbine power output.

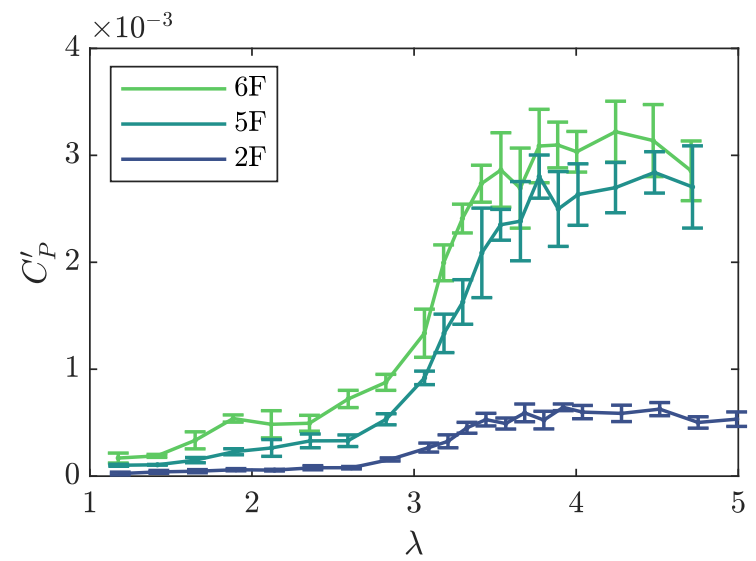

FIG. 17. Power from fluctuating torque and velocity for base flows $2 \mathrm{~F}, 5 \mathrm{~F}$ and $6 \mathrm{~F}$; all confidence intervals included as none is smaller than $1 \%$ of the measurements

As reported in fig. 17, this last component of power is highly affected by the turbine regime. Low values of $\lambda$, corresponding to stalled flow around the blades, result in small fluctuations in power, which rapidly increase once the flow around the blades is attached. It can however be noticed that the contribution of $C_{P}^{\prime}$ is always positive, corresponding to $Q^{\prime}$ and $\omega^{\prime}$ being of the same sign, as confirmed by field data ${ }^{19}$.

From these curves, it can be understood that the main difference between the power curves obtained for constant $\mathcal{I}_{\sigma}$ and varying $\mathcal{T}_{0}$ is on the different stall behaviour of the turbine blades, as reported in fig. 15, with the variations in $\lambda_{\text {peak }}$ and $C_{P}^{\prime}$ being negligible in comparison.

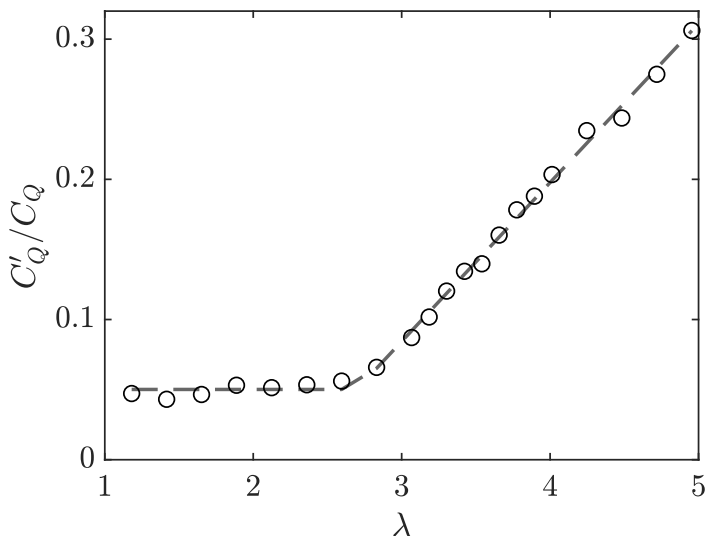

FIG. 18. Sample trend of $C_{Q}^{\prime} / C_{Q}$ for base flow $4 \mathrm{E}$, and ramp fit (dashed line)

While their effect on mean power generation might be negligible, torque fluctuations severely decrease the mean time to failure of full-scale turbines by gearbox fatigue loading, one of the preeminent causes of failure in the field ${ }^{40,41}$. Figure 18 reports the magnitude of torque fluctuations as a percentage of the total torque generated for base flow $4 \mathrm{E}$; for all base flows investigated the ratio $C_{Q}^{\prime} / C_{Q}$ can be reasonably approximated by a ramp function, 


$$
\frac{C_{Q}^{\prime}}{C_{Q}}(\lambda)=C_{1}+C_{2} R\left(\lambda-\lambda_{\text {onset }}\right)
$$

where $C_{1}, C_{2}$ and $\lambda_{\text {onset }}$ are fit coefficients, and $R(x)$ is the ramp function, defined as

$$
R(x)=\max (0, x)
$$

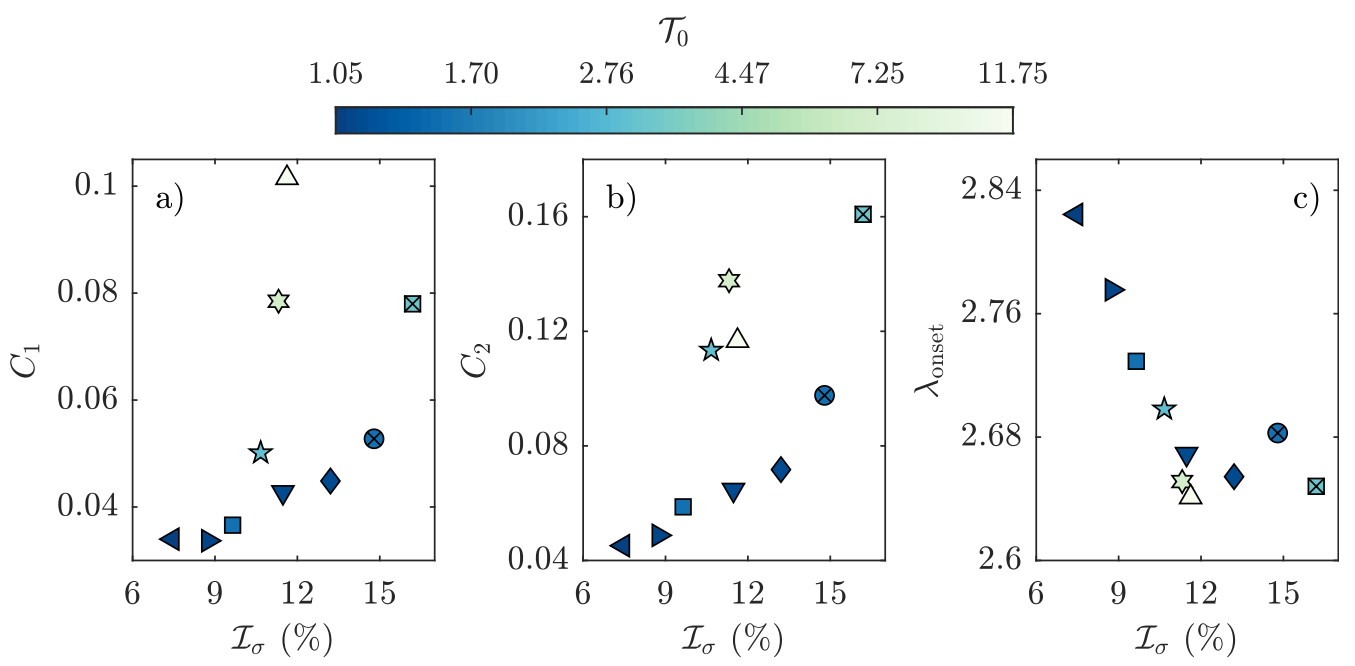

FIG. 19. Dependence of the fit parameters (left to right) $C_{1}, C_{2}$ and $\lambda_{\text {onset }}$ on turbulence characteristics

The effect of turbulence intensity and scale on the baseline torque fluctuations $C_{1}$ is reported in fig. 19a. While there is a limited effect of turbulence intensity on the value of this baseline, it is evident that the main parameter driving the value of $C_{1}$ is the turbulence scale, with the three flows at $\mathcal{I}_{\sigma}=11.5 \%$ resulting in different torque fluctuations, compatibly with the results reported in fig. 17 regarding power extracted from torque fluctuations. A similar trend is observed for the ramp slope $C_{2}$ reported in fig. $19 \mathrm{~b}$, for which a linear increase with turbulence intensity is observed for flows at low $\mathcal{T}_{0}$, and the test cases at high free-stream turbulence scale exhibiting consistently larger values of this parameter.

The value of $\lambda$ that onsets higher torque fluctuations has been reported in fig. 19c; the trend this parameter shows with free-stream turbulence is somewhat similar to what observed for $\lambda_{\text {peak }}$ in fig. 12, with a negligible effect of turbulence scale and a marked linear effect of turbulence intensity until a constant value is kept at $\mathcal{I}_{\sigma}>10.5 \%$.

To summarize the effects of free-stream turbulence on the power extraction mechanisms, power is seen to be positively affected both by flows with large turbulence intensities and long integral time scales; these parameters have effects on the turbine power yield of comparable magnitude, suggesting that neither of these aspects can be overlooked in a realistic forecasting model.

In more detail, free-stream turbulence intensity has a general effect of increasing the turbine power mostly by increasing the torque the individual blades generate for a given regime; this effect is seen for all values of $\lambda$, being the power curves obtained for flows at different $\mathcal{I}_{\sigma}$ visibly staggered for all values of tip-speed ratio (see fig. 10a). The trend of power harvested with turbulence intensity obtained with this model turbine does not follow the customary parabolic behaviour used for large-scale turbines, with the magnitude of power increases being considerably larger than predicted, and an unexpectedly constant power output for $\mathcal{I}_{\sigma}>12 \%$ observed. To explain this behaviour, it can be assumed that the $1+3 \mathcal{I}_{\sigma}^{2}$ term correctly represents the increase in kinetic energy flux through the rotor 
swept area, and any additional power is due to an improvement in the blades performance, observed as an increase in generated torque in fig. 14. This aspect might not be dependent on the low $R e_{c}$ of this present study, as it is also found in data published for aerofoils operating at higher Reynolds numbers ${ }^{38,39,42}$, which might suggest that the parabolic model might underestimate power increases even for full-scale wind turbines. Free-stream turbulence intensity also moderately affects the magnitude of torque fluctuations, albeit in a much smaller amount when compared to turbulence scale, as well as the onset tip-speed ratio of these fluctuations.

Similarly, free-stream integral time scale also has an important effect on the power harvesting mechanisms, with longer time scales translating to a higher power yield for the same turbulence intensity; this is in line with previous findings ${ }^{19,20}$ that have delineated a low-pass behaviour of the wind turbine with respect to incoming velocity fluctuations. The spectra previously reported in fig. $6 \mathrm{~b}$ at constant $\mathcal{I}_{\sigma} \simeq 11.5 \%$ are particularly suited to confirm this finding: despite the same total energy from fluctuations, base flows $5 \mathrm{~F}$ and $6 \mathrm{~F}$ present low-frequency contributions, absent in flow $2 \mathrm{~F}$, that the wind turbine is able to harvest, thus resulting in higher power despite the same turbulence intensity (and thus, the same total energy in the incoming flow). This is reversed at higher frequencies, where flow $2 \mathrm{~F}$ exhibits more energy than the other two; this does not result in a larger power yield as the turbine is not able to convert those fluctuations into usable power. From the data reported in this study and from the analytical modelling presented in ref. 19, it is unclear whether further increasing free-stream integral time scale will keep increasing the wind turbine power output; data from ref. 17 suggests this might not be the case, with increasing $\mathcal{T}_{0}$ resulting in values of power closer to the analytical parabolic trend; however, the low-pass approach of ref. 19 conversely would suggest that increasingly larger values of $\mathcal{T}_{0}$ would generate increasingly higher power until an upper bound, to the point where all the energy in the inflow is contained in large eddies, which the turbine can convert in mechanical power.

It must be noted that the process of power extraction is slightly dependent on the turbine tip-speed ratio $\lambda$, being visibly different between low $\lambda$, where the blades are operating in stalled condition, and high regimes, where the flow around the blades is mostly attached. In particular, it appears that the mean power generated by the turbine is insensitive to the inflow timescales at low tip-speed ratios, with the power curves resulting from the family of flows at constant $\mathcal{I}_{\sigma}$ matching considerably for stalled flow around the blades. This turbine behaviour is unexpected, as previous research in the topic ${ }^{21}$ finds that the turbine low-pass behaviour is not a property of the operating conditions.

This distinction between low- and high- $\lambda$ behaviour of the wind turbine is also observed as an effect on the values of fractional torque unsteadiness $C_{Q}^{\prime} / C_{Q}$, whose values are mostly affected by the free-stream integral time scale. This suggests some independence of the blades aerodynamic properties on the turbulence scale in stalled conditions, possibly mediated by the presence of a large separation bubble around the aerofoils; direct measurements of the velocity and pressure field around the blades in stalled and attached flow conditions are necessary to investigate the causes of this behaviour.

\section{Thrust}

Turbine thrust has been recorded for $\lambda$ ranging from 1 to 5 , and for all base flows reported in table I.

The thrust curves obtained in different base flows appear self-similar, as reported in fig. 20. Unlike the power and torque curves presented in section IIIB, thrust generated by the turbine does not have a clear peak in the $C_{T^{-}} \lambda$ space and, as such, identifying the parameters to normalize $\lambda$ and $C_{T}$ by is more challenging. In this case, we introduce two normalisation coefficients, $k_{\lambda}$ and $k_{T}$, which minimize the least square difference between the reference thrust curve, obtained for base flow $1 \mathrm{~A}$, and the curve $k_{T} C_{T}\left(k_{\lambda} \lambda\right)$ obtained in turbulence. The trends of these parameters as a function of the base flow properties can 


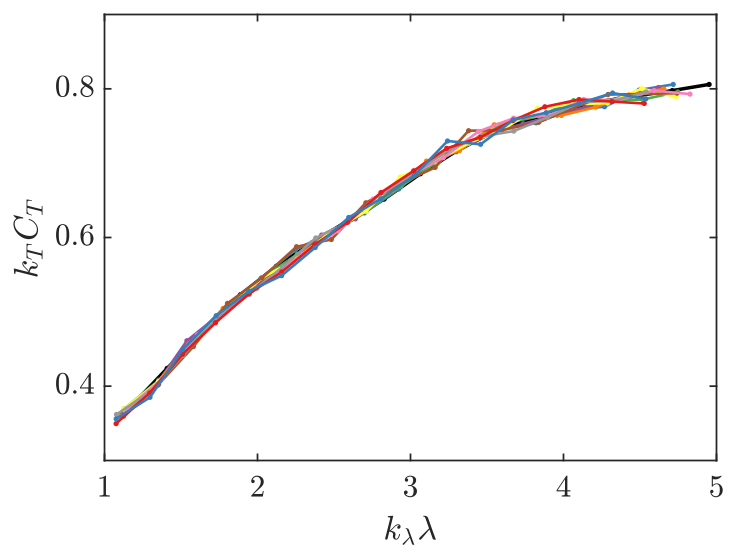

FIG. 20. Self-similarity of thrust curves $C_{T}(\lambda)$

describe the behaviour of the thrust generated by the wind turbine in different operating conditions, which are reported in fig. 21; by definition, the value of both $k_{\lambda}$ and $k_{T}$ for test case $1 \mathrm{~A}$ is 1 .
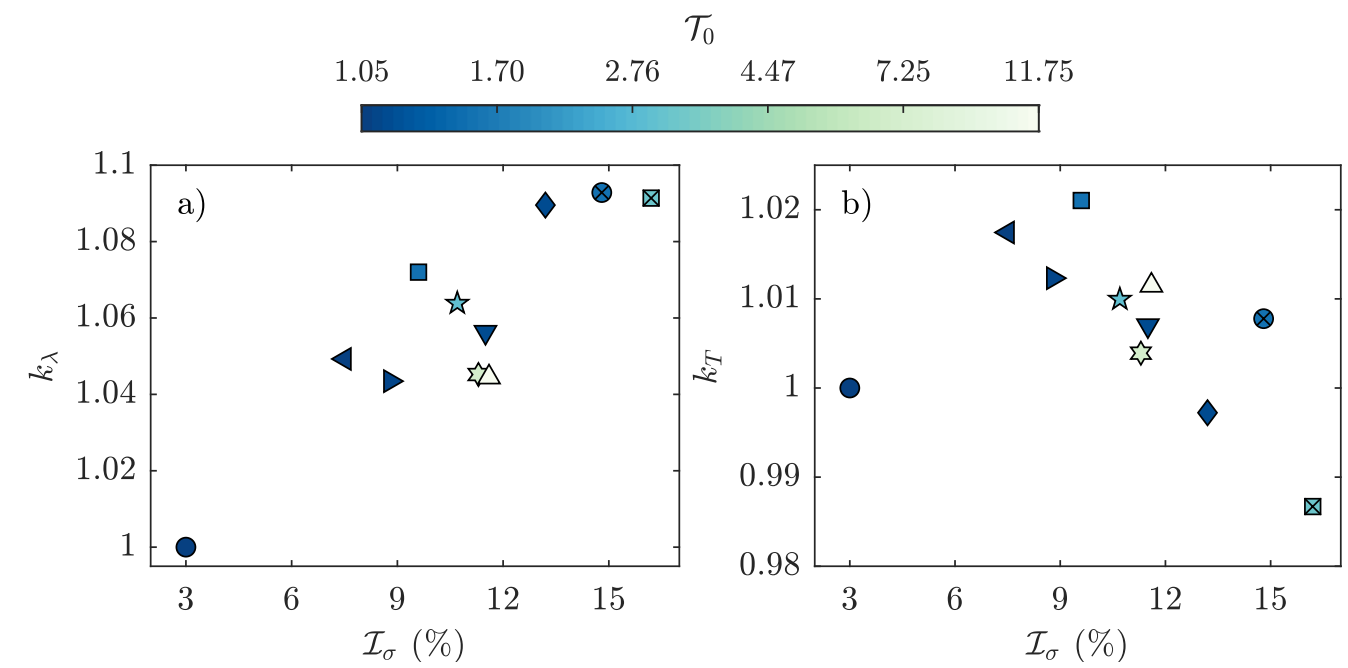

FIG. 21. Trends of $k_{\lambda}$ (left) and $k_{T}$ (right) with turbulence intensity $\mathcal{I}_{\sigma}$ and scale $\mathcal{T}_{0}$

By far, the most evident effect of turbulence on the turbine thrust is the one on $k_{\lambda}$, which is seen broadly and consistently increase with $\mathcal{I}_{\sigma}$, while the effects of $\mathcal{T}_{0}$ are smaller: conceptually, this results on a steeper drag curve for higher values of $\mathcal{I}_{\sigma}$ and thus a larger drag for the same tip-speed ratio. As the effect of $\mathcal{T}_{0}$ on $k_{\lambda}$ is marginal, it would be possible to define a rotor-equivalent velocity that, similarly to what previously presented for power in ref. 8 , is only a function of $\mathcal{I}_{\sigma}$; however, this velocity would not increase as the square of $\mathcal{I}_{\sigma}$, instead reaching an upper bound as the inflow turbulence intensity increases.

Conversely, the effect of $\mathcal{I}_{\sigma}$ on $k_{T}$ appears to be that of a minor increase, possibly concentrated in the range of turbulence intensities between $3 \%$ and $7.5 \%$, followed by a steady decrease, while its values are never exceeding a $2 \%$ difference from the low-turbulence baseline flow.

A behaviour similar to the one observed for $k_{T}$ is also seen on the maximum thrust coefficient reached by the turbine in different base flows, reported in fig. 22: once again, maximum $C_{T}$ decreases steadily with turbulence intensity in the range of $\mathcal{I}_{\sigma}$ here presented, and the total variations are moderate in size, never reaching more than $2 \%$ or less than $3 \%$ of the 

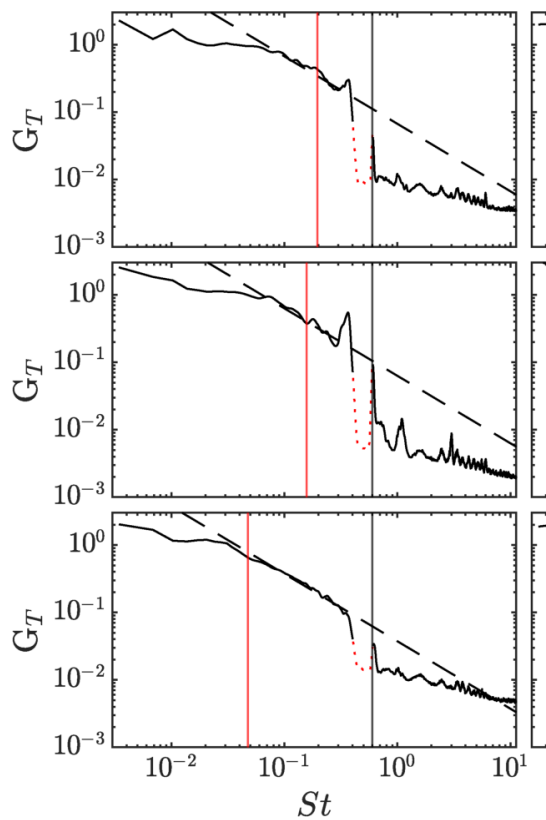
is clear. thrust generated in the low-turbulence test case. However, given that the confidence intervals size is often larger than the difference between consecutive points in fig. 22 , it is difficult to individuate a clear trend, especially for average values of turbulence intensities between $7.5 \%$ and $12 \%$, where a basically constant value of maximum thrust is attained. For values of turbulence intensity larger than $12 \%$, however, the trend of decreasing maximum thrust

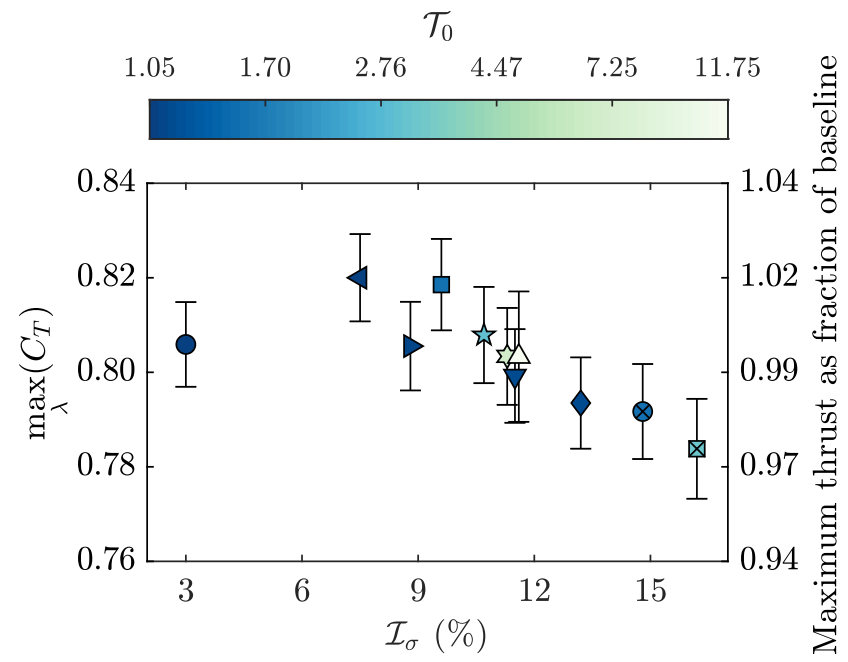

FIG. 22. Dependence of maximum thrust generated on base flows

FIG. 23. Normalised transfer function $G_{T}$ between incoming flow and thrust fluctuations for flows 2B (top), 2F (middle) and 5F (bottom), at tip speed-ratios of $\lambda=1.9$ (left), 3.8 (center) and 4.7 (right); additionally, boundary $S t$ between energy containing and inertial subranges (red vertical line) and turbine reduced angular velocity (black vertical line)

To visualize the effects of velocity fluctuations on thrust unsteadiness, fig. 23 reports the transfer function $\Gamma_{T}$ between incoming flow and unsteady thrust, defined in the frequency 
domain as

$$
\Gamma_{T}(f)=\frac{\phi_{T}(f)}{\phi_{u}(f)}
$$

where $\phi_{T}(f)$ is the power spectral density of the acquired thrust timeseries, as measured by the ATI Mini40 load cell, and $\phi_{u}(f)$ is the spectrum of incoming velocity measured via hot-wire anemometry as described previously in section II C.

The frequency axis is presented as Strouhal number St, defined here as

$$
S t=\frac{f 2 R}{U_{\infty}}
$$

where $2 R$ is the rotor diameter and $U_{\infty}$ is the mean flow velocity during the tests. Normalisation of the vertical axis is performed as

$$
G_{T}=\frac{\Gamma_{T}}{\left(\bar{T} / U_{\infty}\right)^{2}}
$$

where $\bar{T}$ is the time-average thrust for the given tip-speed ratio and base flow, so that $G_{T}$ is adimensional.

In addition to $G_{T}$, the plots also report an estimate of the frequency at which the inertial subrange begins for a given base flow as a red vertical line, the turbine angular velocity as a black vertical line, and the values of $G_{T}$ in the range where the band-stop filter has been applied as dotted red; the best-fitting -1 slope line is also reported in dashed black to each subplot.

The transfer functions are presented for flows $2 \mathrm{~B}, 2 \mathrm{~F}$, and $5 \mathrm{~F}$, thus encompassing low and high values of both $\mathcal{I}_{\sigma}$ and $\mathcal{T}_{0}$; the three tip-speed ratios at which the thrust timeseries has been measured represent a stalled-blade condition $\left(\lambda=1.9\right.$, low $C_{T}$ and $\left.C_{P}\right)$, a peak power $\left(\lambda=3.8\right.$, high $\left.C_{P}\right)$ and a high $C_{T}$ operating condition $(\lambda=4.7)$, thus representing synthetically the different regimes the model wind turbine has been subject to during the experimental campaign.

Similarly to the power transfer functions presented by ref. $19, G_{T}$ is characterized by three distinct regions: a first part of the curve at very low Strouhal numbers, where the turbine response to turbulence is mostly flat; a second region at intermediate frequencies, where the transfer function decreases rapidly following a power law, in this case with exponent -1 ; and a third region for high $S t$ where the transfer function has visibly lower values, up to three orders of magnitude lower than at low frequencies. This overall shape of the thrust transfer function appears to be common between all inflow conditions. Additionally, peaks at the turbine angular velocity along with its higher order harmonics are present for all measurements reported: these can likely be attributed to vibrations generated by the turbine rotor during operation, arising from mechanical unbalance of the turbine, and thus might not be related to a fluid dynamics phenomenon.

While the general shape of $G_{T}$ is comparable with previously published spectra of power frequency response, there are two main differences between power and thrust spectra, namely the slope of the transfer function in its second region, and the extent and bounds of these regions. With regards to the local slope, previously published power transfer functions report a slope of -2 , while from the data here reported it is evident that the best approximating power law has an exponent of -1 : as ref. 20 shows, the -2 slope can be derived from the angular kinetic energy conservation equation; as thrust is not related to this equation, the -2 slope need not apply here.

The bounds of these regions are also different from the ones expected for the power fluctuations transfer function: literature reports the boundary between the first and second region to correspond with the boundary between the energy containing and inertial subranges, which is reported as a red vertical line in fig. 23. From the data reported in this study, it appears that the actual border between region 1 and 2 lies at considerably lower values of $S t$ for the low- $\mathcal{T}_{0}$ flows and at slightly lower values for flow $5 \mathrm{~F}$. A similar result is 
obtained for the critical frequency $f_{c}$ after which the turbine becomes insensitive to the inflow velocity fluctuations: ref. 18 reports this value to be approximately double the turbine angular velocity, while data reported in this study suggests that this is both unaffected by $\lambda$ and always lower than the expected $2 \omega$; no estimation of the actual value of $f_{c}$ can be done from the data here reported as it consistently falls in the range of frequencies removed by the band-stop filter.

While these results are unexpected, they can explain the apparent insensitivity of the turbine thrust on the inflow conditions and simultaneously the high sensitivity of extracted power on the inflow: as the transfer function drops to low values for smaller frequencies, the wind turbine acts as a low-pass filter with a smaller ideal cutoff frequency and thus filters out more of the inflow spectral components. It can be assumed that there exists a range of frequencies for which the turbine cannot convert inflow fluctuations into thrust but can still be used to generate mechanical power.

\section{CONCLUSIONS AND OUTLOOK}

The thrust, power output, and their unsteadiness generated by a model wind turbine have been measured in a wide range of turbulent base flows to identify the trends of these quantities with respect to turbulence parameters and to confirm whether simplified analytical models offer a sufficient degree of accuracy in predicting trends.

Power generated by the model turbine is seen to increase with turbulence intensity, with the magnitude of this increase exceeding what was predicted by ref. 8: a maximum increase of up to $16 \%$ from the low-turbulence baseline has been observed, whereas the simple quadratic model predicted a maximum power increase of $8 \%$. This has been justified as a dependence of the blades aerodynamic properties such as mean torque generated on the free-stream turbulence, thus invalidating the simplifying assumption on which the analytical model is based. From the data obtained in this study it is unclear whether this is due to the low chord-based Reynolds numbers at which the experiments have been carried out; literature however suggests that similar trends hold at higher Reynolds number and a similar trend of power with free-stream turbulence intensity might be observed for full-scale turbines. Free-stream turbulence scale is also positively affecting the turbine power yield; this is in line with previous studies modelling the turbine as a low-pass filter of incoming turbulence, better suited at harvesting power from low-frequency fluctuations.

The ratio between unsteady and mean torque generated by the model turbine can be adequately modelled as a ramp function, constant for low tip-speed ratios and linearly increasing with $\lambda$ at high regimes. Free-stream turbulence scale has been individuated to be the main driving parameter for these fluctuations, with turbulence intensity playing a minor role.

Thrust generated by the model wind turbine is seemingly unaffected by the inflow conditions, with the $C_{T}$ observed in turbulence never differing from the one measured in lowturbulence conditions by more than $2 \%$. The spectra of thrust generated by the turbine have been related to the incoming flow by means of a transfer function, which highlights how high-frequency components in the free-stream are not translated into thrust by the turbine. This behaviour exhibits similarities with results previously published in literature regarding the power generated by turbines of radically different diameters, where the turbine is compared to a low-pass filter; for the case of thrust, the turbine response is seen to be considerably more skewed towards very-low frequencies than for power, which explains the simultaneous high sensitivity of the power harvested and small influence on thrust of the free-stream velocity fluctuations. This behaviour is markedly different from what was observed in literature for bluff bodies like solid plates or turbine simulators such as porous discs, where a clear effect of both turbulence intensity and scale is observed. Results therefore hint at the possibility that these simplified models might not faithfully represent the actual phenomena of thrust-generation, and thus studies employing these simpler geometries might not obtain physically faithful results. 


\section{ACKNOWLEDGMENTS}

This research did not receive any specific grant from funding agencies in the public, commercial or not-for-profit sectors. The PhD scholarship for author SG has been provided by the University of Southampton.

\section{DATA AVAILABILITY STATEMENT}

Data published in this article is available from the University of Southampton repository at https://doi.org/10.5258/SOTON/D1746

\section{REFERENCES}

${ }^{1}$ F. Boettcher, C. Renner, H.-P. Waldl, and J. Peinke, "On the Statistics of Wind Gusts," Bound.-Layer Meteorol. 108, 163-173 (2003).

${ }^{2}$ J. Peinke, S. Barth, F. Böttcher, D. Heinemann, and B. Lange, "Turbulence, a challenging problem for wind energy," Physica A 338, 187-193 (2004).

${ }^{3}$ T. Blackmore, W. Batten, G. Müller, and A. Bahaj, "Influence of turbulence on the drag of solid discs and turbine simulators in a water current," Exp. Fluids 55, 1637 (2014).

${ }^{4}$ S. Aubrun, M. Bastankhah, R. Cal, B. Conan, R. Hearst, D. Hoek, M. Hölling, M. Huang, C. Hur, B. Karlsen, I. Neunaber, M. Obligado, J. Peinke, M. Percin, L. Saetran, P. Schito, B. Schliffke, D. SimsWilliams, O. Uzol, M. Vinnes, and A. Zasso, "Round-robin tests of porous disc models," J. Phys. Conf. Ser. 1256, 012004 (2019).

${ }^{5}$ P. Mycek, B. Gaurier, G. Germain, G. Pinon, and E. Rivoalen, "Experimental study of the turbulence intensity effects on marine current turbines behaviour. Part I: One single turbine," Renew. Energy 66 $729-746$ (2014).

${ }^{6} \mathrm{~W}$. Tian, A. Ozbay, and H. Hu, "A wind tunnel study of wind loads on a model wind turbine in atmospheric boundary layer winds," J. Fluids Struct. 85, 17-26 (2019).

${ }^{7}$ A. Vinod and A. Banerjee, "Performance and near-wake characterization of a tidal current turbine in elevated levels of free stream turbulence," Appl. Energy 254, 113639 (2019).

${ }^{8}$ D. Elliott and J. Cadogan, "Effects of wind shear and turbulence on wind turbine power curves," in 1990 European Community Wind Energy Conference and Exhibition (1990) pp. 1-5.

${ }^{9}$ R. Wagner, I. Antoniou, S. Pedersen, M. Courtney, and H. Jørgensen, "The influence of the wind speed profile on wind turbine performance measurements," Wind Energy 12, 348-362 (2009).

${ }^{10}$ A. Choukulkar, Y. Pichugina, C. Clack, R. Calhoun, R. Banta, A. Brewer, and M. Hardesty, "A new formulation for rotor equivalent wind speed for wind resource assessment and wind power forecasting," Wind Energy 19, 1439-1452 (2016).

${ }^{11} \mathrm{P}$. Bearman and T. Morel, "Effect of free stream turbulence on the flow around bluff bodies," Prog. Aerosp. Sci. 20, 97-123 (1983).

${ }^{12} \mathrm{~L}$. Chamorro and F. Porté-Agel, "A Wind-Tunnel Investigation of Wind-Turbine Wakes: Boundary-Layer Turbulence Effects," Boundary Layer Meteorol. 132, 129-149 (2009).

${ }^{13}$ S. Aubrun, S. Loyer, P. Hancock, and P. Hayden, "Wind turbine wake properties: Comparison between a non-rotating simplified wind turbine model and a rotating model," J. Wind Eng. Ind. Aerodyn. 120, $1-8$ (2013).

${ }^{14}$ L. Lignarolo, D. Ragni, C. Ferreira, and G. van Bussel, "Kinetic energy entrainment in wind turbine and actuator disc wakes: an experimental analysis," J. Phys. Conf. Ser. 524, 012163 (2014).

${ }^{15} \mathrm{G}$. Schubauer and H. Dryden, "The Effect of Turbulence on the Drag of Flat Plates," Tech. Rep. 546 (National Advisory Committe for Aeronautics, Washington D.C., United States, 1935).

${ }^{16} \mathrm{E}$. Rind and I. Castro, "On the effects of free-stream turbulence on axisymmetric disc wakes," Exp. Fluids 53, 301-318 (2012)

${ }^{17}$ Y. Sheinman and A. Rosen, "A dynamic model of the influence of turbulence on the power output of a wind turbine," J. Wind Eng. Ind. Aerodyn. 39, 329-341 (1992).

${ }^{18}$ L. Chamorro, C. Hill, S. Morton, C. Ellis, R. Arndt, and F. Sotiropoulos, "On the interaction between a turbulent open channel flow and an axial-flow turbine," J. Fluid Mech. 716, 658-670 (2013).

${ }^{19}$ L. Chamorro, S. Lee, D. Olsen, C. Milliren, J. Marr, R. Arndt, and F. Sotiropoulos, "Turbulence effects on a full-scale $2.5 \mathrm{MW}$ horizontal-axis wind turbine under neutrally stratified conditions," Wind Energy 18, 339-349 (2014).

${ }^{20}$ N. Tobin, H. Zhu, and L. Chamorro, "Spectral behaviour of the turbulence-driven power fluctuations of wind turbines," J. Turbul. 16, 832-846 (2015).

${ }^{21}$ G. Deskos, G. Payne, B. Gaurier, and M. Graham, "On the spectral behaviour of the turbulence-driven power fluctuations of horizontal-axis turbines," J. Fluid Mech. 904, A13 (2020). 
${ }^{22}$ T. Mücke, D. Kleinhans, and J. Peinke, "Atmospheric turbulence and its influence on the alternating loads on wind turbines," Wind Energy 14, 301-316 (2011).

${ }^{23}$ H. Kadum, S. Rockel, M. Hölling, J. Peinke, and R. B. Cal, "Wind turbine wake intermittency dependence on turbulence intensity and pitch motion," J. Renew. Sustain. Ener. 11, 053302 (2019).

${ }^{24} \mathrm{H}$. Makita, "Realization of a large-scale turbulence field in a small wind tunnel," Fluid Dyn. Res. 8, 53-64 (1991).

${ }^{25}$ R. Hearst and P. Lavoie, "The effect of active grid initial conditions on high Reynolds number turbulence," Exp. Fluids 56, 185 (2015).

${ }^{26}$ R. Poorte and A. Biesheuvel, "Experiments on the motion of gas bubbles in turbulence generated by an active grid," J. Fluid Mech. 461, 127-154 (2002).

${ }^{27}$ E. Dogan, R. E. Hanson, and B. Ganapathisubramani, "Interactions of large-scale free-stream turbulence with turbulent boundary layers," J. Fluid Mech. 802, 79-107 (2016).

${ }^{28} \mathrm{~L}$. Mydlarski and Z. Warhaft, "On the onset of high-Reynolds-number grid-generated wind tunnel turbulence," J. Fluid Mech. 320, 331 (1996).

${ }^{29}$ A. Thormann and C. Meneveau, "Decay of homogeneous, nearly isotropic turbulence behind active fractal grids," Phys. Fluids 26, 025112 (2014).

${ }^{30}$ M. Bastankhah and F. Porté-Agel, "A New Miniature Wind Turbine for Wind Tunnel Experiments. Part I: Design and Performance," Energies 10, 908 (2017).

${ }^{31}$ A. Hyvärinen and A. Segalini, "Effects From Complex Terrain on Wind-Turbine Performance," J. Energy Res. Technol. 139 (2017), 10.1115/1.4036048.

${ }^{32}$ D. Houck and E. Cowen, "A high-accuracy torque transducer for small-scale wind and hydrokinetic turbine experiments," Meas. Sci. Technol. 30, 105005 (2019).

${ }^{33}$ D. Marten, J. Wendler, G. Pechlivanoglou, C. Nayeri, and C. Paschereit, "QBlade: An Open-Source Tool for Design and Simulation of Horizontal and Vertical Axis Wind Turbines," IJETAE 3, 264-269 (2013).

${ }^{34}$ M. Drela, "XFOIL: An Analysis and Design System for Low Reynolds Number Airfoils," in Low Reynolds Number Aerodynamics, Lecture Notes in Engineering, edited by T. J. Mueller (Springer, 1989) pp. 1-12.

${ }^{35}$ L. H. Benedict and R. D. Gould, "Towards better uncertainty estimates for turbulence statistics," Exp. Fluids 22, 129-136 (1996).

${ }^{36}$ A. Abdel-Rahman, C. Tropea, P. Slawson, and A. Strong, "On temperature compensation in hot-wire anemometry," J. Phys. E: Sci. Instrum. 20, 315-319 (1987).

${ }^{37}$ B. Smith, D. Neal, M. Feero, and G. Richards, "Assessing the limitations of effective number of samples for finding the uncertainty of the mean of correlated data," Meas. Sci. Technol. 29, 125304 (2018).

${ }^{38}$ K. Swalwell, J. Sheridan, and W. Melbourne, "The Effect of Turbulence Intensity on Stall of the NACA 0021 Aerofoil," in 14th Australasian Fluid Mechanics Conference (2001) pp. 941-944.

${ }^{39}$ P. Devinant, T. Laverne, and J. Hureau, "Experimental study of wind-turbine airfoil aerodynamics in high turbulence," J. Wind Eng. Ind. Aerodyn. 90, 689-707 (2002).

${ }^{40} \mathrm{~J}$. Ribrant and L. Bertling, "Survey of failures in wind power systems with focus on Swedish wind power plants during 1997-2005," in 2007 IEEE Power Engineering Society General Meeting (2007) pp. 1-8.

${ }^{41}$ P. Tavner, J. Xiang, and F. Spinato, "Reliability analysis for wind turbines," Wind Energy 10, 1-18 (2007).

42 J. Hoffmann, "Effects of freestream turbulence on the performance characteristics of an airfoil," AIAA Journal 29, 1353-1354 (1991). 